\title{
Synthesis and Antimicrobial Evaluation of some New Pyrazole, Pyrazoline and Chromeno[3,4-c]pyrazole Derivatives
}

\author{
Nada M. Abunada, ${ }^{*, a}$ Hamdi M. Hassaneen, ${ }^{b}$ Ahmed S. M. Abu Samaha ${ }^{c}$ and Omar A. Miqdad ${ }^{a}$ \\ ${ }^{a}$ Department of Chemistry and ${ }^{c}$ Department of Biology, Faculty of Applied Sciences, \\ Al-Aqsa University, 76888 Gaza, Palestine \\ ${ }^{b}$ Department of Chemistry, Faculty of Science, Cairo University, Cairo, Egypt
}

\begin{abstract}
Alguns novos derivados de pirazol-5-carbonitrila 8,9 e pirazol-5-carboxamida 13 foram sintetizados pela reação de cicloadição de nitriliminas $\mathbf{3 , 4}$ a $\alpha$-cianocinamonitrilas $\mathbf{5 a - f}$ e $\alpha$-cianocinamamida 12a,b, respectivamente. Por outro lado, a adição de $\mathbf{3 , 4}$ a $\alpha$-cianocinamato de etila 14a-f leva à produção de derivados de 2-pyrazoline-5-carboxilato de etila, 15, 16. Também, a cicloadição de 3,4 à 3-cianocumarina 19a ou à 3 -fenilsulfonilcumarina $19 b$ ou à 3 -bromocumarina 19c leva à produção de derivados do cromeno[3,4-c]pirazol-4(3H)-ona, 20. A cicloadição de $\mathbf{3 , 4}$ à 3-acetilcumarina, 22 e 3-benzoilcumarina, 23, produz o correspondente diidrocromeno[3,4-c] pirazol-4(3H)-ona, 24 e 25, respectivamente. A oxidação de 24 e 25 produz 20 . A maioria dos compostos preparados mostrou boa a moderada atividade antibacteriana e antifúngica.
\end{abstract}

Some new pyrazole-5-carbonitrile derivatives 8,9 and pyrazole-5-carboxamide 13 were synthesized by the cycloaddition reaction of nitrilimines $\mathbf{3 , 4}$ to $\alpha$-cyanocinnamonitriles $\mathbf{5 a - f}$ and $\alpha$-cyanocinnamamide 12a,b respectively. On the other hand 3,4 add to ethyl $\alpha$-cyanocinnamate 14a-f to give ethyl 2-pyrazoline-5-carboxylate derivatives 15,16 . Also, cycloaddition of 3,4 to 3-cyanocoumarin 19a or 3-phenylsulphonylcoumarin 19b or 3-bromocoumarin 19c give chromeno[3,4-c]pyrazol-4(3H)-one derivatives 20. In the same direction, the cycloaddition of $\mathbf{3 , 4}$ to 3-acetylcoumarin $\mathbf{2 2}$ and 3-benzoylcoumarin $\mathbf{2 3}$ gives the corresponding dihydrochromeno[3,4-c] pyrazol-4(3H)-one 24 and 25 respectively. Oxidation of $\mathbf{2 4}$ and 25 give $\mathbf{2 0}$. Most of the prepared compounds showed good to moderate antibacterial and antifungal activities.

Keywords: nitrilimines, pyrazole, pyrazoline, chromeno[3,4-c]pyrazole

\section{Introduction}

Pyrazole and heterocyclic fused pyrazole derivatives represent an important class of heterocyclic compounds that have many applications. Some of these compounds are employed as anti-inflammatory compounds,${ }^{1-3}$ as blood platelet aggregation inhibitors, ${ }^{1}$ as adenosine antagonists, ${ }^{4,5}$ and as controlling herbicides. ${ }^{6}$ They also show antimicrobial and antiparasitic activities. ${ }^{7,8}$ Also, pyrazoline derivatives have been found to possess antifungal, ${ }^{9}$ antidepressant ${ }^{10-13}$ anticonvulsant, ${ }^{12,13}$ antiinflammatory, ${ }^{14}$ antibacterial ${ }^{15}$ and anti-tumor ${ }^{16}$ activities. Chromenopyrazoles exhibit high activity against gram positive and gram negative bacteria. ${ }^{17}$ Moreover, many selectively fluoro-substituted organic compounds show a peculiar pharmacological and

*e-mail: nadanadannrs@yahoo.com agrochemical properties. ${ }^{18-23}$ Therefore, as a connection of our interest in the chemistry of the preparation of heterocyclic compounds from hydrazonoyl halides ${ }^{24-29}$ and the above-mentioned findings, the present work is aimed at the preparation of new pyrazole, pyrazoline and their chromene fused derivatives incorporating fluorine and chlorine substituents into these derivatives hoping that it would potentiate their expected biological activities.

\section{Experimental}

Hydrazonoyl bromides $\mathbf{1}^{30}$ and $\mathbf{2}^{31}$ were prepared by known methods. Melting points were measured on electrothermal melting point apparatus and are uncorrected. Elemental analyses were carried out at the Microanalytical Center of Cairo University, Giza, Egypt. Infrared spectra 
were recorded in potassium bromide pellets on a Pye Unicam SP 3-300 and Shimadzu FT-IR 8101 PC infrared spectrophotometer. NMR spectra were recorded at 200 $\left({ }^{1} \mathrm{H}\right)$ and $50\left({ }^{13} \mathrm{C}\right) \mathrm{MHz}$ in (DMSO-d6) on a GEMINI-200 spectrometer. Chemical shifts $(\delta)$ are reported relative to TMS as the internal standard. Mass spectra were measured on a GCMS-QP 1000 EX spectrometer operating at an ionization potential of $70 \mathrm{eV}$.

Synthesis of 3,4-diaryl-1-(4-nitrophenyl)-1H-pyrazole-5carbonitriles 8,9

Triethylamine $(0.7 \mathrm{~mL}, 5 \mathrm{mmol})$ was added to a stirred solution of the appropriate hydrazonoyl bromides 1,2 and the appropriate $\alpha$-cyanocinnamonitrile derivatives $\mathbf{5 a - f}$ $(5 \mathrm{mmol})$ in benzene $(40 \mathrm{~mL})$ at room temperature. The mixture was refluxed for $8 \mathrm{~h}$ as indicated by TLC. The precipitated triethylamine hydrobromide was removed by filtration and the filtrate was evaporated, and then triturated with methanol. The solid that formed was collected by filtration and crystallized from the suitable solvent to give 8,9 respectively.

\section{3-(4-Fluorophenyl)-1-(4-nitrophenyl)-4-phenyl-1H-} pyrazole-5-carbonitrile $8 \boldsymbol{a}$

Obtained as pale yellow crystals; yield: $1.07 \mathrm{~g}(56 \%)$; $\mathrm{mp} 214-6{ }^{\circ} \mathrm{C}$ (from dioxane-ethanol); IR (KBr) $v_{\max } / \mathrm{cm}^{-1}$ : 3119.0, 3083.1 (CH-aromatic), $2235.5(\mathrm{C} \equiv \mathrm{N}), 1659.2$ $(\mathrm{C}=\mathrm{N}), 1592.7(\mathrm{C}=\mathrm{C}) ;{ }^{1} \mathrm{H}$ NMR $\left(\mathrm{DMSO}-\mathrm{d}_{6}\right) \delta$ 7.04-8.54 (m, 13H, ArH's); ${ }^{13} \mathrm{C}$ NMR (DMSO-d $\left.{ }_{6}\right) \delta 161.28$ (d, J 256.4 $\mathrm{Hz}, \mathrm{C}-\mathrm{F}), 146.38,146.14$ (C=N, C-p- $\mathrm{NO}_{2}$ ), 143.10, 138.83, $135.12,129.76$ (d, $J 8.3 \mathrm{~Hz}, \mathrm{C}-m-\mathrm{F}), 129.25,127.97$, $125.72,126.52,125.14,124.86$ (d, J3.1 Hz, C- $p$-F), 124.71, $115.50(\mathrm{~d}, J 22.1 \mathrm{~Hz}, \mathrm{C}-o-\mathrm{F}), 110.61(\mathrm{C} \equiv \mathrm{N}) ; \mathrm{MS}, m / z: 385$ $\left(\mathrm{M}^{+}+1,88.8\right), 384\left(\mathrm{M}^{+}, 100.0\right), 338$ (10.3), 337 (15.0), 196 (10.4), 75 (14.5), 63 (13.4). Anal. Calc. for $\mathrm{C}_{22} \mathrm{H}_{13} \mathrm{FN}_{4} \mathrm{O}_{2}$ $\left(\mathrm{M}_{\mathrm{r}}=384.36\right): \mathrm{C}, 68.74 ; \mathrm{H}, 3.40 ; \mathrm{N}, 14.57 \%$; Found: $\mathrm{C}$, 68.62; H, 3.39; N, 14.34\%.

3-(2,4-Dichlorophenyl)-1-(4-nitrophenyl)-4-phenyl-1Hpyrazole-5-carbonitrile 9 a

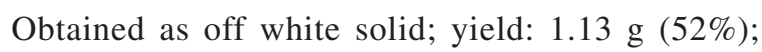
mp $160-162{ }^{\circ} \mathrm{C}$ (from acetic acid); IR (KBr) $v_{\max } / \mathrm{cm}^{-1}$ : $3085.3(\mathrm{CH}$-aromatic), $2228.1(\mathrm{C} \equiv \mathrm{N}), 1652.5(\mathrm{C}=\mathrm{N})$, $1595.1(\mathrm{C}=\mathrm{C}) ;{ }^{1} \mathrm{H}$ NMR (DMSO-d $\left.\mathrm{d}_{6}\right) \delta$ 7.01-8.54 (m, $12 \mathrm{H}$, ArH's); ${ }^{13} \mathrm{C}$ NMR (DMSO-d ${ }_{6}$ ) $\delta 147.30,146.38$ $\left(\mathrm{C}=\mathrm{N}, \mathrm{C}-p-\mathrm{NO}_{2}\right), 145.14,138.83,135.12,133.36,132.45$, $130.33,130.08,129.98,129.45,129.25,127.97,125.72$, $126.52,125.14,124.71,110.61(\mathrm{C} \equiv \mathrm{N}) ; \mathrm{MS}, m / z: 436$ $\left(\mathrm{M}^{+}+2,46.3\right), 434\left(\mathrm{M}^{+}, 68.1\right), 401$ (38.4), 399 (100.0), 355 (16.4), 353 (48.3), 319 (6.3), 317 (18.7), 246 (17.0),
216 (15.0), 190 (18.6), 127 (23.5), 75 (53.1), 50 (39.2). Anal. Calc. for $\mathrm{C}_{22} \mathrm{H}_{12} \mathrm{Cl}_{2} \mathrm{~N}_{4} \mathrm{O}_{2}\left(\mathrm{M}_{\mathrm{r}}=435.26\right): \mathrm{C}, 60.70$; $\mathrm{H}, 2.77 ; \mathrm{N}, 12.87 ; \mathrm{Cl}, 16.29 \%$; Found: C, 60.58; H, 2.87; $\mathrm{N}, 12.69 ; \mathrm{Cl}, 16.31 \%$.

3-(4-Fluorophenyl)-4-(4-methylphenyl)-1-(4-nitrophenyl)1H-pyrazole-5-carbonitrile $8 \boldsymbol{b}$

Obtained as off white solid; yield: $1.01 \mathrm{~g}(51 \%) ; \mathrm{mp}$ $163-165^{\circ} \mathrm{C}$ (from acetic acid); $\mathrm{IR}(\mathrm{KBr}) v_{\max } / \mathrm{cm}^{-1}: 3116.1$, 3080.6 (CH-aromatic), 2924.1, 2854.2 (CH-aliphatic), $2233.1(\mathrm{C} \equiv \mathrm{N}), 1650.6(\mathrm{C}=\mathrm{N}), 1597.0(\mathrm{C}=\mathrm{C}) ;{ }^{1} \mathrm{H}$ NMR $\left(\right.$ DMSO-d $\left._{6}\right) \delta$ 7.02-8.58 (m, 12H, ArH's), 2.25 (s, 3H, $\mathrm{CH}_{3}$ ); ${ }^{13} \mathrm{C}$ NMR (DMSO-d $) \delta 161.30$ (d, J $256.4 \mathrm{~Hz}, \mathrm{C}-\mathrm{F}$ ), $146.39,146.10\left(\mathrm{C}=\mathrm{N}, \mathrm{C}-p-\mathrm{NO}_{2}\right), 143.13,138.77,135.09$, 129.78 (d, J 8.3 Hz, C-m-F), 129.28, 128.48, 126.92, $126.51,125.22,124.88$ (d, J $3.1 \mathrm{~Hz}, \mathrm{C}-p-\mathrm{F}), 122.85,115.52$ $(\mathrm{d}, J 22.1 \mathrm{~Hz}, \mathrm{C}-o-\mathrm{F}), 110.58(\mathrm{C} \equiv \mathrm{N}), 20.39\left(\mathrm{CH}_{3}\right)$; MS, m/z: $399\left(\mathrm{M}^{+}+1,71.4\right), 398\left(\mathrm{M}^{+}, 100.0\right), 383$ (13.5), 351 (10.8), 76 (14.9), 75 (23.2). Anal. Calc. for $\mathrm{C}_{23} \mathrm{H}_{15} \mathrm{FN}_{4} \mathrm{O}_{2}$ $\left(\mathrm{M}_{\mathrm{r}}=398.38\right): \mathrm{C}, 69.34 ; \mathrm{H}, 3.79 ; \mathrm{N}, 14.06 \%$; Found: $\mathrm{C}$, $69.14 ; \mathrm{H}, 3.82 ; \mathrm{N}, 13.96 \%$.

3-(2,4-Dichlorophenyl)-4-(4-methylphenyl)-1-(4nitrophenyl)-1H-pyrazole-5-carbonitrile $9 \boldsymbol{b}$

Obtained as off white solid; yield: $1.16 \mathrm{~g}(52 \%) ; \mathrm{mp}$ $195-197{ }^{\circ} \mathrm{C}$ (from acetic acid); IR (KBr) $v_{\max } / \mathrm{cm}^{-1}: 3081.2$ (CH-aromatic), 2920.3, 2854.8 (CH-aliphatic), 2226.9 $(\mathrm{C} \equiv \mathrm{N}), 1653.1(\mathrm{C}=\mathrm{N}), 1595.0(\mathrm{C}=\mathrm{C}) ;{ }^{1} \mathrm{H}$ NMR $\left(\mathrm{DMSO}_{6}\right)$ $\delta$ 7.02-8.54 (m, 11H, ArH's), 2.26 (s, 3H, $\left.\mathrm{CH}_{3}\right) ;{ }^{13} \mathrm{C} \mathrm{NMR}$ $\left(\right.$ DMSO-d $\left.{ }_{6}\right) \delta 147.32,146.39\left(\mathrm{C}=\mathrm{N}, \mathrm{C}-\mathrm{p}-\mathrm{NO}_{2}\right), 146.10$, 138.77, 135.09, 133.40, 132.44, 130.30, 130.10, 129.96, $129.43,129.28,128.48,126.92,126.51,125.22,122.85$ (15C, ArC's), $110.58(\mathrm{C} \equiv \mathrm{N}), 20.36\left(\mathrm{CH}_{3}\right) ; \mathrm{MS}, \mathrm{m} / z: 450$ $\left(\mathrm{M}^{+}+2,76.8\right), 448\left(\mathrm{M}^{+}, 100.0\right), 413(82.8), 378(48.1), 367$ (42.6), 257 (10.9), 230 (12.6), 165 (11.2), 140 (27.8), 103 (14.7), 90 (21.7), 75 (49.1). Anal. Calc. for $\mathrm{C}_{23} \mathrm{H}_{14} \mathrm{Cl}_{2} \mathrm{~N}_{4} \mathrm{O}_{2}$ $\left(\mathrm{M}_{\mathrm{r}}=449.28\right): \mathrm{C}, 61.48 ; \mathrm{H}, 3.14 ; \mathrm{N}, 12.47 ; \mathrm{Cl}, 15.78 \%$; Found: C, 61.42; H, 3.21; N, 12.39; Cl, 15.69\%.

3-(4-Fluorophenyl)-4-(4-methoxyphenyl)-1-(4nitrophenyl)-1H-pyrazole-5-carbonitrile $8 c$

Obtained as off white solid; yield: $1.01 \mathrm{~g}(49 \%)$; $\mathrm{mp} 194-196{ }^{\circ} \mathrm{C}$ (from acetic acid); IR (KBr) $\mathrm{v}_{\max } / \mathrm{cm}^{-1}$ : 3120.7, 3089.4 (CH-aromatic), 2936.5 (CH-aliphatic), $2231.1(\mathrm{C} \equiv \mathrm{N}), 1651.8(\mathrm{C}=\mathrm{N}), 1598.6(\mathrm{C}=\mathrm{C}) ;{ }^{1} \mathrm{H}$ NMR $\left(\right.$ DMSO-d $\left._{6}\right) \delta$ 7.03-8.58 (m, 12H, ArH's), 3.77 (s, 3H, $\left.\mathrm{OCH}_{3}\right) ;{ }^{13} \mathrm{C}$ NMR $\left(\mathrm{DMSO}-\mathrm{d}_{6}\right) \delta 161.31(\mathrm{~d}, J 256.4 \mathrm{~Hz}$, $\mathrm{C}-\mathrm{F}), 160.82\left(\mathrm{C}-\mathrm{OCH}_{3}\right), 146.39,146.10\left(\mathrm{C}=\mathrm{N}, \mathrm{C}-\mathrm{p}-\mathrm{NO}_{2}\right)$, 143.12, 135.11, 129.78 (d, $J 8.3 \mathrm{~Hz}, \mathrm{C}-m-\mathrm{F}), 129.67$, $129.27,126.51,125.22,124.86$ (d, J 3.1 Hz, C-p-F), 116.23, 115.52 (d, $J 22.1 \mathrm{~Hz}, \mathrm{C}-o-\mathrm{F}), 114.54,110.58$ 
$(\mathrm{C} \equiv \mathrm{N}), 55.18\left(\mathrm{OCH}_{3}\right) ; \mathrm{MS}, \mathrm{m} / z: 414\left(\mathrm{M}^{+}, 100.0\right), 399$ (9.3), 75 (19.8), 74 (17.9). Anal. Calc. for $\mathrm{C}_{23} \mathrm{H}_{15} \mathrm{FN}_{4} \mathrm{O}_{3}$ $\left(\mathrm{M}_{\mathrm{r}}=414.38\right): \mathrm{C}, 66.66 ; \mathrm{H}, 3.64 ; \mathrm{N}, 13.52 \%$; Found: $\mathrm{C}$, $66.43 ; \mathrm{H}, 3.61 ; \mathrm{N}, 13.49 \%$.

3-(2,4-Dichlorophenyl)-4-(4-methoxyphenyl)-1-(4nitrophenyl)-1H-pyrazole-5-carbonitrile $9 \boldsymbol{c}$

Obtained as off white solid; yield: $1.11 \mathrm{~g}(48 \%) ; \mathrm{mp}$ $147-149{ }^{\circ} \mathrm{C}$ (from acetic acid); IR (KBr) $v_{\max } / \mathrm{cm}^{-1}: 3082.6$ (CH-aromatic), 2933.8 (CH-aliphatic), $2224.6(\mathrm{C} \equiv \mathrm{N})$, 1652.4 $(\mathrm{C}=\mathrm{N}), 1596.6(\mathrm{C}=\mathrm{C}) ;{ }^{1} \mathrm{H}$ NMR (DMSO) $\delta$ 7.01$8.55\left(\mathrm{~m}, 11 \mathrm{H}, \mathrm{ArH}\right.$ 's), $3.78\left(\mathrm{~s}, 3 \mathrm{H}, \mathrm{OCH}_{3}\right) ;{ }^{13} \mathrm{C} \mathrm{NMR}$ $\left(\mathrm{DMSO}_{-} \mathrm{d}_{6}\right) \delta 159.33\left(\mathrm{C}-\mathrm{OCH}_{3}\right), 148.02,146.50(\mathrm{C}=\mathrm{N}$, C- $\left.p-\mathrm{NO}_{2}\right), 141.91,134.69,133.41,133.21,131.43,129.00$, 128.84, 128.43, 127.39, 124.94, 122.99, 119.88, 114.20, 112.15 (14C, ArC's), $110.78(\mathrm{C} \equiv \mathrm{N}), 54.75\left(\mathrm{OCH}_{3}\right)$; MS, $m / z: 466\left(\mathrm{M}^{+}+2,62.1\right), 464\left(\mathrm{M}^{+}, 100.0\right), 394$ (17.5), 76 (18.2), 75 (41.7), 63 (17.4), 62 (16.4), 50 (17.7). Anal. Calc. for $\mathrm{C}_{23} \mathrm{H}_{14} \mathrm{Cl}_{2} \mathrm{~N}_{4} \mathrm{O}_{3}\left(\mathrm{M}_{\mathrm{r}}=465.28\right)$ : $\mathrm{C}, 59.37 ; \mathrm{H}, 3.03$; N, 12.04; Cl, 15.23\%; Found: C, 59.15; H, 3.11; N, 12.14; $\mathrm{Cl}, 15.31 \%$.

4-(4-Chlorophenyl)-3-(4-fluorophenyl)-1-(4-nitrophenyl)1H-pyrazole-5-carbonitrile $8 d$

Obtained as off white solid; yield: $1.06 \mathrm{~g} \mathrm{(51 \% );} \mathrm{mp}$ $160-2{ }^{\circ} \mathrm{C}$ (from acetic acid); IR (KBr) $v_{\max } / \mathrm{cm}^{-1}: 3113.3$, 3079.5 (CH-aromatic), $2231.6(\mathrm{C} \equiv \mathrm{N}), 1656.3(\mathrm{C}=\mathrm{N})$, $1594.6(\mathrm{C}=\mathrm{C}) ;{ }^{1} \mathrm{H}$ NMR $\left(\right.$ DMSO-d $\left._{6}\right) \delta$ 7.03-8.54 (m, $12 \mathrm{H}$, ArH's); ${ }^{13} \mathrm{C}$ NMR (DMSO-d $) \delta 161.33$ (d, $J 256.4 \mathrm{~Hz}$, C-F), $146.42,146.16\left(\mathrm{C}=\mathrm{N}, \mathrm{C}-p-\mathrm{NO}_{2}\right), 143.18,137.55$, 135.11, 130.09, 129.76 (d, $J 8.3 \mathrm{~Hz}, \mathrm{C}-m-\mathrm{F}), 128.88$, $126.89,126.50,125.20,124.88$ (d, J3.1 Hz, C- $p$-F), 121.05, $115.53(\mathrm{~d}, J 22.1 \mathrm{~Hz}, \mathrm{C}-o-\mathrm{F}), 110.61(\mathrm{C} \equiv \mathrm{N}) ; \mathrm{MS}, m / z: 419$ $\left(\mathrm{M}^{+}+1,84.1\right), 418\left(\mathrm{M}^{+}, 100.0\right), 371(10.9), 338$ (12.4), 230 (12.6), 95 (10.7), 75 (26.0), 63 (15.7), 50 (19.2). Anal. Calc. for $\mathrm{C}_{22} \mathrm{H}_{12} \mathrm{ClFN}_{4} \mathrm{O}_{2}\left(\mathrm{M}_{\mathrm{r}}=418.80\right)$ : C, 63.09; H, 2.88; N, 13.37; Cl, 8.46\%; Found: C, 62.89; H, 2.94; N, 13.29; $\mathrm{Cl}, 8.52 \%$.

4-(4-Chlorophenyl)-3-(2,4-dichlorophenyl)-1-(4nitrophenyl)-1H-pyrazole-5-carbonitrile $9 d$

Obtained as white solid; yield: $1.10 \mathrm{~g}(47 \%) ; \mathrm{mp}$ $168-170^{\circ} \mathrm{C}$ (from acetic acid); IR (KBr) $v_{\max } / \mathrm{cm}^{-1}: 3087.7$ (CH-aromatic), $2228.7(\mathrm{C} \equiv \mathrm{N}), 1655.3(\mathrm{C}=\mathrm{N}), 1596.6$ $(\mathrm{C}=\mathrm{C}) ;{ }^{1} \mathrm{H}$ NMR (DMSO-d $\left.{ }_{6}\right) \delta$ 7.32-8.56 (m, 11H, ArH's); ${ }^{13} \mathrm{C}$ NMR $\left(\mathrm{DMSO}-\mathrm{d}_{6}\right) \delta 148.08,146.42\left(\mathrm{C}=\mathrm{N}, \mathrm{C}-p-\mathrm{NO}_{2}\right)$, 146.16, 137.55, 135.11, 133.45, 132.67, 131.87, 130.62, $130.09,129.87,129.74,128.88,126.89,126.50,125.20$, 121.05 (15C, ArC's), $110.61(\mathrm{C} \equiv \mathrm{N}) ; \mathrm{MS}, m / z: 470\left(\mathrm{M}^{+}+2\right.$, 100.0), 468 ( $\left.\mathrm{M}^{+}, 92.1\right), 433$ (56.1), 400 (36.8), 387 (41.9), 386 (21.3), 317 (28.1), 289 (19.4), 277 (45.5), 216 (28.9),
177 (22.1), 163 (20.9), 136 (23.3), 100 (26.9), 90 (22.5), 75 (89.7), 63 (83.0), 51 (41.5), 50 (96.8). Anal. Calc. for $\mathrm{C}_{22} \mathrm{H}_{11} \mathrm{Cl}_{3} \mathrm{~N}_{4} \mathrm{O}_{2}\left(\mathrm{M}_{\mathrm{r}}=469.70\right): \mathrm{C}, 56.25 ; \mathrm{H}, 2.36 ; \mathrm{N}$, 11.92; Cl, 22.64\%; Found: C, 56.29; H, 2.35; N, 11.94; $\mathrm{Cl}, 22.66 \%$.

\section{3,4-Di-(4-fluorophenyl)-1-(4-nitrophenyl)-1H-pyrazole- 5-carbonitrile $8 \boldsymbol{e}$}

Obtained as yellow crystals; yield: $0.96 \mathrm{~g} \mathrm{(48 \% );} \mathrm{mp}$ $178-180{ }^{\circ} \mathrm{C}$ (from acetic acid); IR (KBr) $v_{\max } / \mathrm{cm}^{-1}: 3114.6$, $3079.9(\mathrm{CH}$-aromatic), $2231.8(\mathrm{C} \equiv \mathrm{N}), 1657.1(\mathrm{C}=\mathrm{N})$, $1595.1(\mathrm{C}=\mathrm{C}) ;{ }^{1} \mathrm{H}$ NMR $\left(\right.$ DMSO-d $\left._{6}\right) \delta$ 7.04-8.58 (m, 12H, ArH's); ${ }^{13} \mathrm{C}$ NMR (DMSO-d $\left.{ }_{6}\right) \delta 164.40(\mathrm{~d}, J 255.8 \mathrm{~Hz}$, C-F), 161.30 (d, J 256.4 Hz, C-F), 146.39, 146.10 (C=N, C- $p$ - $\mathrm{NO}_{2}$ ), 143.13, 135.09, 132.74 (d, J 8.2 Hz, C-m-F), $129.78(\mathrm{~d}, J 8.3 \mathrm{~Hz}, \mathrm{C}-m-\mathrm{F}), 129.2,126.51,125.22,124.88$ (d, J 3.1 Hz, C-p-F), 123.72 (d, J 3.2 Hz, C-p-F), 116.89 (d, J $22.4 \mathrm{~Hz}, \mathrm{C}-o-\mathrm{F}), 115.52$ (d, J $22.1 \mathrm{~Hz}, \mathrm{C}-o-\mathrm{F}), 110.58$ $(\mathrm{C} \equiv \mathrm{N}) ; \mathrm{MS}, \mathrm{m} / \mathrm{z}: 403\left(\mathrm{M}^{+}+1,98.3\right), 402\left(\mathrm{M}^{+}, 100.0\right), 356$ (10.0), 355 (12.6), 214 (11.9), 75 (19.6). Anal. Calc. for $\mathrm{C}_{22} \mathrm{H}_{12} \mathrm{~F}_{2} \mathrm{~N}_{4} \mathrm{O}_{2}\left(\mathrm{M}_{\mathrm{r}}=402.35\right): \mathrm{C}, 65.67 ; \mathrm{H}, 3.00 ; \mathrm{N}, 13.92 \%$; Found: C, 65.46; H, 2.96; N, 13.87\%.

3-(2,4-Dichlorophenyl)-4-(4-fluorophenyl)-1-(4nitrophenyl)-1H-pyrazole-5-carbonitrile $9 e$

Obtained as off white solid; yield: $1.04 \mathrm{~g} \mathrm{(46 \% );} \mathrm{mp}$ $170-172{ }^{\circ} \mathrm{C}$ (from acetic acid); IR (KBr) $v_{\max } / \mathrm{cm}^{-1}: 3113.3$, $3087.4(\mathrm{CH}$-aromatic), $2228.1(\mathrm{C} \equiv \mathrm{N}), 1655.9(\mathrm{C}=\mathrm{N})$, $1596.4(\mathrm{C}=\mathrm{C}) ;{ }^{1} \mathrm{H}$ NMR (DMSO-d $\left.{ }_{6}\right) \delta$ 7.31-8.59 (m, $11 \mathrm{H}$, ArH's); ${ }^{13} \mathrm{C}$ NMR (DMSO-d $) \delta 164.40(\mathrm{~d}, J 255.8 \mathrm{~Hz}$, C-F), 148.47, $146.39,146.10\left(\mathrm{C}=\mathrm{N}, \mathrm{C}-p-\mathrm{NO}_{2}\right), 135.09$, 133,65, 132.46, 132.74 (d, J 8.2 Hz, C-m-F), 130.45, $130.21,129.88,129.48,129.2,126.51,125.22,123.72$ (d, J 3.2 Hz, C-p-F), 115.52 (d, J 22.1 Hz, C-o-F), 110.58 $(\mathrm{C} \equiv \mathrm{N}) ; \mathrm{MS}, \mathrm{m} / z: 454\left(\mathrm{M}^{+}+2,80.8\right), 452(\mathrm{M}+, 100.0), 417$ (63.0), 371 (46.8), 335 (16.2), 264 (31.8), 208 (37.0), 145 (35.2), 107 (30.5), 75 (54.7), 63 (45.3), 50 (51.0). Anal. Calc. for $\mathrm{C}_{22} \mathrm{H}_{11} \mathrm{Cl}_{2} \mathrm{FN}_{4} \mathrm{O}_{2}\left(\mathrm{M}_{\mathrm{r}}=453.25\right)$ : $\mathrm{C}, 58.29 ; \mathrm{H}, 2.44$; $\mathrm{N}, 12.36$; Cl, 15.64\%; Found: C, 58.31; H, 2.46; N, 11.34; $\mathrm{Cl}, 15.66 \%$.

3-(2,4-Dichlorophenyl)-1,4-di-(4-nitrophenyl)- $1 \mathrm{H}$ pyrazole-5-carbonitrile $9 \boldsymbol{f}$

Obtained as brown crystals; yield: $1.05 \mathrm{~g}(44 \%)$; mp 220-222 ${ }^{\circ} \mathrm{C}$ (from acetic acid); IR (KBr) $v_{\max } / \mathrm{cm}^{-1}$ : 3086.9 (CH-aromatic), $2233.7(\mathrm{C} \equiv \mathrm{N}), 1594.8(\mathrm{C}=\mathrm{C}) ;{ }^{1} \mathrm{H}$ NMR (DMSO-d $\left.{ }_{6}\right) \delta 7.58-8.56$ (m, 11H, ArH's); ${ }^{13} \mathrm{C}$ NMR $\left(\right.$ DMSO-d $\left._{6}\right) \delta 148.22,147.37,146.46\left(\mathrm{C}=\mathrm{N}, 2 \mathrm{C}-p-\mathrm{NO}_{2}\right)$, 145.24, 135.56, 134.02, 133.89, 131.15, 130.31, 130.16, $129.94,129.38,128.64,128.34,126.78,125.42,119.78$ (14C, ArC's), $110.61(\mathrm{C} \equiv \mathrm{N})$; MS, $m / z: 481\left(\mathrm{M}^{+}+2,80.8\right)$, 
$479\left(\mathrm{M}^{+}, 100.0\right), 398$ (92.3), 351 (21.8), 317 (18.5), 215 (21.5), 76 (28.1), 75 (38.1), 63 (29.6), 50 (37.2). Anal. Calc. for $\mathrm{C}_{22} \mathrm{H}_{11} \mathrm{Cl}_{2} \mathrm{~N}_{5} \mathrm{O}_{4}\left(\mathrm{M}_{\mathrm{r}}=480.25\right)$ : $\mathrm{C}, 55.01 ; \mathrm{H}, 2.47$; N, 14.64; Cl, 14.76\%; Found: C, 55.00; H, 2.46; N, 14.65; $\mathrm{Cl}, 14.77 \%$.

Synthesis of 3,4-diaryl-1-(4-nitrophenyl)-1H-pyrazole-5carboxamides 13

This reaction was carried out by the same method described for the preparation of the previous pyrazoles $\mathbf{8 , 9}$ using $\alpha$-cyanocinnamamide derivatives $\mathbf{1 2 a}, \mathbf{b}$ in place of $\alpha$-cyanocinnamonitrile derivatives 5a-f. The prepared compounds 13a,b together with their physical and spectral data are listed below.

3-(2,4-Dichlorophenyl)-1-(4-nitrophenyl)-4-phenyl-1Hpyrazole-5-carboxamide $\mathbf{1 3 a}$

Obtained as off white solid; yield: $1.04 \mathrm{~g}(46 \%)$; mp 233-235 ${ }^{\circ} \mathrm{C}$ (from acetic acid); IR (KBr) $v_{\max } / \mathrm{cm}^{-1}$ : 3354.9, $3254.1\left(\mathrm{NH}_{2}\right), 3083.4(\mathrm{CH}$-aromatic), 1669.6 $(\mathrm{C}=\mathrm{O}$ amide $), 1627.2(\mathrm{C}=\mathrm{N}), 1597.0(\mathrm{C}=\mathrm{C}) ;{ }^{1} \mathrm{H}$ NMR $\left(\right.$ DMSO-d $\left._{6}\right) \delta$ 7.18-8.46 (m, 14H, ArH's, $\left.\mathrm{NH}_{2}\right) ;{ }^{13} \mathrm{C}$ NMR $\left(\mathrm{DMSO}_{-} \mathrm{d}_{6}\right) \delta 167.78(\mathrm{C}=\mathrm{O}$ amide $), 149.38,145.63(\mathrm{C}=\mathrm{N}$, C- $\left.p-\mathrm{NO}_{2}\right), 145.12,133.38,132.39,130.03,129.82$, 129.77, 128.39, 127.78, 127.67, 127.02, 126.69, 125.09, 124.87, 124.38, 123.78 (15C, ArC's); MS, m/z: 454 $\left(\mathrm{M}^{+}+2,65.1\right), 452\left(\mathrm{M}^{+}, 95.4\right), 400$ (77.8), 354 (51.6), 356 (25.8), 351 (33.7), 190 (26.6), 168 (19.8), 163 (21.2), 115 (26.2), 90 (22.0), 89 (100.0), 76 (36.6), 63 (33.9), 50 (34.5). Anal. Calc. for $\mathrm{C}_{22} \mathrm{H}_{14} \mathrm{Cl}_{2} \mathrm{~N}_{4} \mathrm{O}_{3}\left(\mathrm{M}_{\mathrm{r}}=453.27\right)$ : $\mathrm{C}$, 58.29; H, 3.11; N, 12.36; Cl, 15.64\%; Found: C, 58.31; $\mathrm{H}, 3.12 ; \mathrm{N}, 12.38 ; \mathrm{Cl}, 15.66 \%$.

\section{3-(2,4-Dichlorophenyl)-1,4-di-(4-nitrophenyl)- $1 \mathrm{H}$ - pyrazole-5-carboxamide $\mathbf{1 3 b}$}

Obtained as pale yellow solid; yield: $1.12 \mathrm{~g} \mathrm{(45 \% );}$ mp $182-184{ }^{\circ} \mathrm{C}$ (from acetic acid); IR (KBr) $v_{\max } / \mathrm{cm}^{-1}$ : 3301.4, 3228.6 ( $\left.\mathrm{NH}_{2}\right), 3117.5,3092.1$ (CH-aromatic), $1711.0(\mathrm{C}=\mathrm{O}$ amide $), 1591.0(\mathrm{C}=\mathrm{C}) ;{ }^{1} \mathrm{H}$ NMR (DMSO-d $\left.{ }_{6}\right)$ $\delta$ 7.42-8.52 (m, 11H, ArH's), 6.25 (s, br., $\left.2 \mathrm{H}, \mathrm{NH}_{2}\right) ;{ }^{13} \mathrm{C}$ NMR (DMSO-d ${ }_{6}$ ) $\delta 167.77$ (C=O amide), 149.72, 145.61 $\left(\mathrm{C}=\mathrm{N}, \mathrm{C}-\mathrm{p}-\mathrm{NO}_{2}\right), 145.40,136.16,133.38,132.32,129.82$, $129.79,128.39,127.76,127.53,127.09,126.31,126.23$, 124.89, 124.37, 121.78 (15C, ArC's), $21.38\left(\mathrm{CH}_{3}\right)$; MS, $m / z: 499\left(\mathrm{M}^{+}+2,71.5\right), 497\left(\mathrm{M}^{+}, 100.0\right), 462$ (12.6), 418 (13.5), 416 (30.5), 362 (16.9), 360 (12.4), 190 (16.0), 88 (11.3), 76 (19.3), 63 (14.4), 50 (17.1). Anal. Calc. for $\mathrm{C}_{22} \mathrm{H}_{13} \mathrm{Cl}_{2} \mathrm{~N}_{5} \mathrm{O}_{5}\left(\mathrm{M}_{\mathrm{r}}=498.27\right): \mathrm{C}, 53.02 ; \mathrm{H}, 2.62 ; \mathrm{N}$, $14.05 ; \mathrm{Cl}, 14.23 \%$; Found: C, 53.00; H, 2.64; N, 14.07; $\mathrm{Cl}, 14.22 \%$.
Synthesis of ethyl 5-cyano-3,4-diaryl-1-(4-nitrophenyl)-2pyrazoline-5-carboxylates 15,16

This reaction was carried out by the same method described for the preparation of the previous pyrazoles $\mathbf{8 , 9}$ using ethyl $\alpha$-cyanocinnamate derivatives $14 a-f$ in place of $\alpha$-cyanocinnamonitrile derivatives 5a-f. Compounds 15,16 with their physical and spectral data are listed below.

Ethyl 5-cyano-3-(4-fluorophenyl)-1-(4-nitrophenyl)-4phenyl-2-pyrazoline-5-carboxylate 15a

Obtained as yellow crystals; yield: $1.19 \mathrm{~g}(52 \%)$; mp 214-215 ${ }^{\circ} \mathrm{C}$ (from acetic acid); IR (KBr) $v_{\max } / \mathrm{cm}^{-1}$. 3107.7, 3088.4 (CH-aromatic), 2984.3 (CH-aliphatic), $1762.6\left(\mathrm{C}=\mathrm{O}\right.$ ester), $1592.9(\mathrm{C}=\mathrm{C}) ;{ }^{1} \mathrm{H}$ NMR $\left(\right.$ DMSO- $\left._{6}\right)$ $\delta$ 7.13-8.42 (m, 13H, ArH's), 6.20 (s, 1H pyrazoline), 4.08 (q, $2 \mathrm{H}, J 7.0 \mathrm{~Hz}, \mathrm{COOCH}{ }_{2} \mathrm{CH}_{3}$ ), 0.93 (t, $3 \mathrm{H}, J 7.0$ $\left.\mathrm{Hz}, \mathrm{COOCH}_{2} \mathrm{CH}_{3}\right) ;{ }^{13} \mathrm{C}$ NMR (DMSO-d $\left.)_{6}\right) 165.17(\mathrm{~d}$, $J 256.1 \mathrm{~Hz}, \mathrm{C}-\mathrm{F}), 164.38$ (C=O ester), 148.26, 146.25 $\left(\mathrm{C}=\mathrm{N}, \mathrm{C}-p-\mathrm{NO}_{2}\right), 143.43,132.65,130.40,129.37,129.20$ (d, J 8.3 Hz, C-m-F), 127.25 (d, J 3.1 Hz, C-p-F), 127.04, 124.73, 120.97, 115.97 (d, J 22.2 Hz, C-o-F), 111.02 $(\mathrm{C} \equiv \mathrm{N}), 70.92$ (C-5 pyrazoline), 63.35 (C-4 pyrazoline), $61.31\left(\mathrm{OCH}_{2} \mathrm{CH}_{3}\right), 12.91\left(\mathrm{OCH}_{2} \mathrm{CH}_{3}\right) ; \mathrm{MS}, \mathrm{m} / z: 459$ $\left(\mathrm{M}^{+}+1,17.4\right), 458\left(\mathrm{M}^{+}, 17.0\right), 386$ (100.0), 385 (94.0), 340 (21.2), 339 (21.9), 90 (5.3), 76 (6.6), (21.9), 90 (5.3), 76 (6.6), 51 (4.5), 50 (4.6). Anal. Calc. for $\mathrm{C}_{25} \mathrm{H}_{19} \mathrm{FN}_{4} \mathrm{O}_{4}$ $\left(\mathrm{M}_{\mathrm{r}}=458.44\right): \mathrm{C}, 65.49 ; \mathrm{H}, 4.17 ; \mathrm{N}, 12.22$; Found: $\mathrm{C}$, $65.56 ; \mathrm{H}, 4.26 ; \mathrm{N}, 12.45 \%$.

Ethyl 5-cyano-3-(2,4-dichlorophenyl)-1-(4-nitrophenyl)4-phenyl-2-pyrazoline-5-carboxylate 16 a

Obtained as yellow crystals; yield: $1.29 \mathrm{~g} \mathrm{(51 \% );} \mathrm{mp}$ 203-204 ${ }^{\circ} \mathrm{C}$ (from acetic acid); IR (KBr) $v_{\max } / \mathrm{cm}^{-1}: 3066.3$ (CH-aromatic), 2995.8, 2926.0 (CH-aliphatic), 1742.7 $(\mathrm{C}=\mathrm{O}$ ester $), 1655.0(\mathrm{C}=\mathrm{N}), 1592.6(\mathrm{C}=\mathrm{C}) ;{ }^{1} \mathrm{H}$ NMR $\left(\right.$ DMSO-d $\left._{6}\right) \delta$ 7.12-8.43 (m, 12H, ArH's), $6.21(\mathrm{~s}, 1 \mathrm{H}$ pyrazoline), 4.17 (q, $\left.2 \mathrm{H}, J 7.2 \mathrm{~Hz}, \mathrm{COOCH}{ }_{2} \mathrm{CH}_{3}\right), 0.95$ $\left(\mathrm{t}, 3 \mathrm{H}, J 7.2 \mathrm{~Hz}, \mathrm{COOCH}_{2} \mathrm{CH}_{3}\right) ;{ }^{13} \mathrm{C}$ NMR $\left(\mathrm{DMSO}_{6} \mathrm{~d}_{6}\right)$ $\delta 163.39(\mathrm{C}=\mathrm{O}$ ester $), 148.58,146.13\left(\mathrm{C}=\mathrm{N}, \mathrm{C}-\mathrm{p}-\mathrm{NO}_{2}\right)$, 143.83, 134.05, 133.82, 133.26, 132.48, 130.37, 129.47, 129.17, 128.25, 127.08, 126.88, 124.96, 121.78 (13C, ArC's), 112.98 (C $\equiv N), 70.71$ (C-5 pyrazoline), 64.25 (C-4 pyrazoline), $61.24\left(\mathrm{OCH}_{2} \mathrm{CH}_{3}\right), 12.84\left(\mathrm{OCH}_{2} \mathrm{CH}_{3}\right)$; MS, $m / z: 510\left(\mathrm{M}^{+}+2,9.8\right), 508\left(\mathrm{M}^{+}, 11.2\right), 437$ (62.4), 435 (100.0), 391 (14.0), 389 (24.7), 219 (8.4), 218 (11.0), 190 (10.4), 90 (15.0), 89 (15.9), 77 (17.0), 76 (20.9), 75 (12.9), 63 (19.0), 51 (15.9), 50 (16.6). Anal. Calc. for $\mathrm{C}_{25} \mathrm{H}_{18} \mathrm{Cl}_{2} \mathrm{~N}_{4} \mathrm{O}_{4}\left(\mathrm{M}_{\mathrm{r}}=509.34\right): \mathrm{C}, 58.95 ; \mathrm{H}, 3.56 ; \mathrm{N}$, 10.99 ; Cl, 13.92\%; Found: C, 58.83; H, 3.58; N, 11.02; $\mathrm{Cl}, 14.01 \%$. 
Ethyl 5-cyano-3-(4-fluorophenyl)-4-(4-methylphenyl)-1-(4nitrophenyl)-2-pyrazoline-5-carboxylate $\mathbf{1 5 b}$

Obtained as yellow crystals; yield: $1.27 \mathrm{~g} \mathrm{(54 \% );} \mathrm{mp}$ $187-190{ }^{\circ} \mathrm{C}$ (from acetic acid); IR (KBr) $v_{\max } / \mathrm{cm}^{-1}: 3188.7$ (CH-aromatic), 2983.3, 2961.1 (CH-aliphatic), 1747.1 $\left(\mathrm{C}=\mathrm{O}\right.$ ester), $1589.0(\mathrm{C}=\mathrm{C}) ;{ }^{1} \mathrm{H}$ NMR $\left(\right.$ DMSO- $\left._{6}\right) \delta 7.14$ 8.44 (m, 12H, ArH's), 6.21 (s, 1H pyrazoline), 4.07 (q, 2H, $\left.J 7.0 \mathrm{~Hz}, \mathrm{COOCH}_{2} \mathrm{CH}_{3}\right), 2.26\left(\mathrm{~s}, 3 \mathrm{H}, \mathrm{CH}_{3}\right), 0.91(\mathrm{t}, 3 \mathrm{H}$, $J 7.0 \mathrm{~Hz}, \mathrm{COOCH}_{2} \mathrm{CH}_{3}$ ); ${ }^{13} \mathrm{C}$ NMR (DMSO-d $\left.{ }_{6}\right) \delta 165.17$ (d, $J 256.1 \mathrm{~Hz}, \mathrm{C}-\mathrm{F}$ ), 164.38 (C=O ester), $148.26,146.25$ $\left(\mathrm{C}=\mathrm{N}, \mathrm{C}-p-\mathrm{NO}_{2}\right), 143.43,137.86,130.88,129.20(\mathrm{~d}, J 8.3$ Hz, C-m-F), 128.16, 127.25 (d, J 3.1 Hz, C-p-F), 126.54, 124.73, 120.99, 115.97 (d, J 22.2 Hz, C-o-F), 111.02 $(\mathrm{C} \equiv \mathrm{N}), 70.92$ (C-5 pyrazoline), 63.35 (C-4 pyrazoline), $61.31\left(\mathrm{OCH}_{2} \mathrm{CH}_{3}\right), 21.06\left(\mathrm{CH}_{3}\right), 12.91\left(\mathrm{OCH}_{2} \mathrm{CH}_{3}\right) ; \mathrm{MS}$, $\mathrm{m} / \mathrm{z}: 473\left(\mathrm{M}^{+}+1,11.7\right), 472\left(\mathrm{M}^{+}, 13.8\right), 400$ (89.4), 399 (100.0), 354 (17.6), 353 (20.8), 76 (12.3), 63 (10.7). Anal. Calc. for $\mathrm{C}_{26} \mathrm{H}_{21} \mathrm{FN}_{4} \mathrm{O}_{4}\left(\mathrm{M}_{\mathrm{r}}=472.46\right): \mathrm{C}, 66.09 ; \mathrm{H}, 4.48 ; \mathrm{N}$, $11.85 \%$; Found: C, 65.89; H, 4.39; N, 11.76\%.

Ethyl 5-cyano-3-(2,4-dichlorophenyl)-4-(4-methylphenyl)1-(4-nitrophenyl)-2-pyrazoline-5-carboxylate $\mathbf{1 6 b}$

Obtained as yellow crystals; yield: $1.36 \mathrm{~g}(52 \%)$; mp 200-203 ${ }^{\circ} \mathrm{C}$ (from acetic acid); IR (KBr) $v_{\max } / \mathrm{cm}^{-1}: 3063.7$ (CH-aromatic), 2982.3 (CH-aliphatic), 1761.1 (C=O ester), $1651.8(\mathrm{C}=\mathrm{N})$; $^{1} \mathrm{H}$ NMR (DMSO-d $\left.{ }_{6}\right) \delta$ 7.11-8.42 (m, 11H, ArH's), 6.21 (s, 1H, pyrazoline), 4.11-4.21 (q, 2H, J 7.2 $\left.\mathrm{Hz}, \mathrm{COOCH} \mathrm{CH}_{3}\right), 2.27\left(\mathrm{~s}, 3 \mathrm{H}, \mathrm{CH}_{3}\right), 0.98(\mathrm{t}, 3 \mathrm{H}, J 7.2$ $\mathrm{Hz}, \mathrm{COOCH}_{2} \mathrm{CH}_{3}$ ); ${ }^{13} \mathrm{C}$ NMR (DMSO-d $\left.)_{6}\right) \delta 163.37(\mathrm{C}=\mathrm{O}$ ester), 148.61, $146.15\left(\mathrm{C}=\mathrm{N}, \mathrm{C}-p-\mathrm{NO}_{2}\right), 143.86,135.66$, 134.10, 133.28, 132.45, 130.89, 130.34, 129.48, 129.16, 127.03, 126.82, 124.96, 121.81 (13C, ArC's), 112.86 $(\mathrm{C} \equiv \mathrm{N}), 70.82$ (C-5 pyrazoline), 64.31 (C-4 pyrazoline), $61.27\left(\mathrm{OCH}_{2} \mathrm{CH}_{3}\right), 21.34\left(\mathrm{CH}_{3}\right), 12.84\left(\mathrm{OCH}_{2} \underline{\mathrm{CH}}_{3}\right) ; \mathrm{MS}$, $\mathrm{m} / \mathrm{z}: 522\left(\mathrm{M}^{+}, 14.1\right), 451$ (81.4), 449 (100.0), 403 (18.0), 76 (18.0), 75 (15.9), 63 (13.9), 62 (13.3), 50 (12.2). Anal. Calc. for $\mathrm{C}_{26} \mathrm{H}_{20} \mathrm{Cl}_{2} \mathrm{~N}_{4} \mathrm{O}_{4}\left(\mathrm{M}_{\mathrm{r}}=523.36\right)$ : C, 59.66; H, 3.85; N, $10.70 ; \mathrm{Cl}, 13.54 \%$; Found: C, 59.58; H, 3.82; N, 10.68; $\mathrm{Cl}, 13.49 \%$.

Ethyl 5-cyano-3-(4-fluorophenyl)-4-(4-methoxyphenyl)-1(4-nitrophenyl)-2-pyrazoline-5-carboxylate 15c

Obtained as yellow crystals; yield: $1.19 \mathrm{~g}$ (49\%); mp $183-184{ }^{\circ} \mathrm{C}$ (from acetic acid); IR (KBr) $v_{\max } / \mathrm{cm}^{-1}: 3076.8$ (CH-aromatic), 2983.3 (CH-aliphatic), $1764.5(\mathrm{C}=\mathrm{O}$ ester), 1597.7 (C=C); ${ }^{1} \mathrm{H}$ NMR (DMSO-d ${ }_{6}$ ) $\delta$ 7.14-8.43 (m, 12H, ArH's), 6.20 (s, 1H pyrazoline), 4.08 (q, 2H, $J$ $\left.7.2 \mathrm{~Hz}, \mathrm{COOCH}_{2} \mathrm{CH}_{3}\right), 3.76$ (s, $\left.3 \mathrm{H}, \mathrm{OCH}_{3}\right), 0.90$ (t, $3 \mathrm{H}, J$ $\left.7.2 \mathrm{~Hz}, \mathrm{COOCH}_{2} \underline{\mathrm{CH}}_{3}\right) ;{ }^{13} \mathrm{C} \mathrm{NMR}\left(\mathrm{DMSO}-\mathrm{d}_{6}\right) \delta 165.17(\mathrm{~d}$, $J 256.1 \mathrm{~Hz}, \mathrm{C}-\mathrm{F}), 164.38$ (C=O ester), $158.10\left(\mathrm{C}-\mathrm{OCH}_{3}\right)$, 148.26, $146.25\left(\mathrm{C}=\mathrm{N}, \mathrm{C}-p-\mathrm{NO}_{2}\right), 143.43,129.20$ (d, J 8.3
Hz, C-m-F), 128.23, 127.25 (d, J $3.1 \mathrm{~Hz}, \mathrm{C}-p-\mathrm{F}), 126.54$, 124.73, 123.17, 121.10, 115.97 (d, J 22.2 Hz, C-o-F), 111.02 $(\mathrm{C} \equiv \mathrm{N}), 70.92$ (C-5 pyrazoline), 63.35 (C-4 pyrazoline), 61.31 $\left(\mathrm{OCH}_{2} \mathrm{CH}_{3}\right), 54.40\left(\mathrm{OCH}_{3}\right), 12.91\left(\mathrm{OCH}_{2} \underline{\mathrm{CH}}_{3}\right) ; \mathrm{MS}, \mathrm{m} / z$ : $489\left(\mathrm{M}^{+}+1,5.4\right), 488\left(\mathrm{M}^{+}, 13.1\right), 416(91.5), 415$ (100.0), 370 (4.1), 369 (14.2), 77 (10.9), 75 (9.6). Anal. Calc. for $\mathrm{C}_{26} \mathrm{H}_{21} \mathrm{FN}_{4} \mathrm{O}_{5}\left(\mathrm{M}_{\mathrm{r}}=488.46\right): \mathrm{C}, 63.92 ; \mathrm{H}, 4.33 ; \mathrm{N}, 11.47 \%$; Found: C, 63.79; H, 4.26; N, $11.41 \%$.

Ethyl 5-cyano-3-(2,4-dichlorophenyl)-4-(4methoxyphenyl)-1-(4-nitrophenyl)-2-pyrazoline-5carboxylate $16 \mathrm{c}$

Obtained as yellow crystals; yield: $1.26 \mathrm{~g}(47 \%)$; $\mathrm{mp} 182-184{ }^{\circ} \mathrm{C}$ (from acetic acid); IR (KBr) $v_{\max } / \mathrm{cm}^{-1}$ : 3071.8 (CH-aromatic), 2974.7 (CH-aliphatic), 1763.4 $(\mathrm{C}=\mathrm{O}$ ester $), 1651.1 \quad(\mathrm{C}=\mathrm{N}), 1591.7(\mathrm{C}=\mathrm{C}) ;{ }^{1} \mathrm{H}$ NMR (DMSO-d $\mathrm{d}_{6}$ ) $\delta$ 7.13-8.44 (m, 11H, ArH's), 6.23 (s, 1H pyrazoline), 4.18 (q, $2 \mathrm{H}, J 7.2 \mathrm{~Hz}, \mathrm{COOCH}_{2} \mathrm{CH}_{3}$ ), 3.77 (s, $\left.3 \mathrm{H}, \mathrm{OCH}_{3}\right), 0.96\left(\mathrm{t}, 3 \mathrm{H}, J 7.2 \mathrm{~Hz}, \mathrm{COOCH}_{2} \mathrm{CH}_{3}\right) ;{ }^{13} \mathrm{C} \mathrm{NMR}$ $\left(\right.$ DMSO-d $\left.{ }_{6}\right) \delta 163.39(\mathrm{C}=\mathrm{O}$ ester $), 158.79^{2}\left(\mathrm{C}-\mathrm{OCH}_{3}\right)$, $148.58,146.13\left(\mathrm{C}=\mathrm{N}, \mathrm{C}-\mathrm{p}-\mathrm{NO}_{2}\right), 143.83,134.05,133.26$, 132.48, 130.37, 129.47, 128.63, 126.88, 126.49, 124.96, 124.03, 121.78 (12C, ArC's), $112.98(\mathrm{C} \equiv \mathrm{N}), 70.71(\mathrm{C}-5$ pyrazoline), 64.25 (C-4 pyrazoline), $61.24\left(\mathrm{OCH}_{2} \mathrm{CH}_{3}\right)$, $54.50\left(\mathrm{OCH}_{3}\right), 12.84\left(\mathrm{OCH}_{2} \mathrm{CH}_{3}\right) ; \mathrm{MS}, m / z: 540\left(\mathrm{M}^{+}+2\right.$, 10.9), $538\left(\mathrm{M}^{+}, 14.1\right), 467$ (70.1), 465 (100.0), 421 (8.2), 419 (13.0), 77 (8.4), 76 (11.1), 63 (8.8), 50 (7.4). Anal. Calc. for $\mathrm{C}_{26} \mathrm{H}_{20} \mathrm{Cl}_{2} \mathrm{~N}_{4} \mathrm{O}_{5}\left(\mathrm{M}_{\mathrm{r}}=539.36\right)$ : C, 57.89, H, 3.73; N, 10.38; Cl, 13.14\%; Found: C, 57.70; H, 3.78; N, 10.29; $\mathrm{Cl}, 13.09 \%$.

Ethyl 4-(4-chlorophenyl)-5-cyano-3-(4-fluorophenyl)-1-(4nitrophenyl)-2-pyrazoline-5-carboxylate 15d

Obtained as yellow crystals; yield: $1.15 \mathrm{~g} \mathrm{(47 \% );} \mathrm{mp}$ $202-203^{\circ} \mathrm{C}$ (from acetic acid); IR (KBr) $v_{\text {max }} / \mathrm{cm}^{-1}: 3078.7$ (CH-aromatic), 2981.4 (CH-aliphatic), 1758.7 (C=O ester), $1589.0(\mathrm{C}=\mathrm{C}) ;{ }^{1} \mathrm{H}$ NMR $\left(\right.$ DMSO-d $\left._{6}\right) \delta$ 7.13-8.42 (m, 12H, ArH's), 6.34 (s, 1H pyrazoline), 4.01 (q, 2H, J 7.1 $\mathrm{Hz}, \mathrm{COOCH}{ }_{2} \mathrm{CH}_{3}$ ), 0.90 (t, $3 \mathrm{H}, J 7.1 \mathrm{~Hz}, \mathrm{COOCH}_{2} \mathrm{CH}_{3}$ ); ${ }^{13} \mathrm{CNMR}$ (DMSO-d $) \delta 165.17$ (d, J $\left.256.1 \mathrm{~Hz}, \mathrm{C}-\mathrm{F}\right), 164.38$ $(\mathrm{C}=\mathrm{O}$ ester $), 148.26,146.25\left(\mathrm{C}=\mathrm{N}, \mathrm{C}-\mathrm{p}-\mathrm{NO}_{2}\right), 143.43$, 133.08, 131.82, 131.29, 129.20 (d, $J 8.3 \mathrm{~Hz}, \mathrm{C}-m-\mathrm{F})$, 128.48, 127.25 (d, J 3.1 Hz, C-p-F), 124.73, 120.98 , 115.97 (d, $J 22.2 \mathrm{~Hz}, \mathrm{C}-o-\mathrm{F}), 111.03(\mathrm{C} \equiv \mathrm{N}), 70.92(\mathrm{C}-5$ pyrazoline), 63.35 (C-4 pyrazoline), $61.31\left(\mathrm{OCH}_{2} \mathrm{CH}_{3}\right)$, $12.91\left(\mathrm{OCH}_{2} \mathrm{CH}_{3}\right) ; \mathrm{MS}, \mathrm{m} / z: 493\left(\mathrm{M}^{+}+1,10.1\right), 492\left(\mathrm{M}^{+}\right.$, 11.5), 420 (88.8), 419 (100.0), 374 (24.5), 373 (25.9), 217 (9.8), 216 (13.3), 122 (13.1), 76 (24.7), 50 (23.0). Anal. Calc. for $\mathrm{C}_{25} \mathrm{H}_{18} \mathrm{ClFN}_{4} \mathrm{O}_{4}\left(\mathrm{M}_{\mathrm{r}}=492.88\right)$ : $\mathrm{C}, 60.91 ; \mathrm{H}, 3.68$; N, 11.36; Cl, 7.19\%; Found: C, 60.87; H, 3.66; N, 11.41; $\mathrm{Cl}, 7.23 \%$. 
Ethyl 4-(4-chlorophenyl)-5-cyano-3-(2,4-dichlorophenyl)1-(4-nitrophenyl)-2-pyrazoline-5-carboxylate 16d

Obtained as yellow crystals; yield: $1.38 \mathrm{~g}(51 \%) ; \mathrm{mp}$ 202-204 ${ }^{\circ} \mathrm{C}$ (from acetic acid); IR (KBr) $v_{\max } / \mathrm{cm}^{-1}: 3064.3$ (CH-aromatic), 2983.2 (CH-aliphatic), $1760.2(\mathrm{C}=\mathrm{O}$ ester), $1652.2(\mathrm{C}=\mathrm{N}), 1591.1(\mathrm{C}=\mathrm{C}) ;{ }^{1} \mathrm{H}$ NMR $\left(\mathrm{DMSO}_{\mathrm{d}}\right)$ $\delta$ 7.14-8.43 (m, 11H, ArH's), 6.23 (s, 1H pyrazoline), 4.18 (q, $\left.2 \mathrm{H}, J 7.2 \mathrm{~Hz}, \mathrm{COOCH}_{2} \mathrm{CH}_{3}\right), 0.98(\mathrm{t}, 3 \mathrm{H}, J 7.2 \mathrm{~Hz}$, $\left.\mathrm{COOCH}_{2} \mathrm{CH}_{3}\right) ;{ }^{13} \mathrm{C}$ NMR (DMSO-d 6 ) $\delta 163.41$ (C=O ester), $148.55,146.11\left(\mathrm{C}=\mathrm{N}, \mathrm{C}-p-\mathrm{NO}_{2}\right), 143.84,134.05,133.68$, 133.26, 132.48, 131.54, 131.28, 130.37, 129.47, 128.50, 126.88, 124.96, 121.78 (13C, ArC's), $112.88(\mathrm{C} \equiv \mathrm{N})$, 70.70 (C-5 pyrazoline), 64.27 (C-4 pyrazoline), 61.22 $\left(\mathrm{OCH}_{2} \mathrm{CH}_{3}\right), 12.83\left(\mathrm{OCH}_{2} \underline{\mathrm{CH}}_{3}\right) ; \mathrm{MS}, \mathrm{m} / z: 546\left(\mathrm{M}^{+}+4,3.9\right)$, $544\left(\mathrm{M}^{+}+2,12.5\right), 542\left(\mathrm{M}^{+}, 10.8\right), 473$ (34.9), 471 (100.0), 469 (98.2), 427 (5.1), 425 (17.0), 423 (18.0), 76 (11.0), 75 (11.3), 63 (8.9), 50 (9.9). Anal. Calc. for $\mathrm{C}_{25} \mathrm{H}_{17} \mathrm{Cl}_{3} \mathrm{~N}_{4} \mathrm{O}_{4}$ $\left(\mathrm{M}_{\mathrm{r}}=543.78\right): \mathrm{C}, 55.21 ; \mathrm{H}, 3.15 ; \mathrm{N}, 10.30 ; \mathrm{Cl}, 19.55 \%$; Found: C, 55.23; H, 3.21; N, 10.19; Cl, 19.48\%.

Ethyl 5-cyano-3,4-di-(4-fluorophenyl)-1-(4-nitrophenyl)2-pyrazoline-5-carboxylate 15e

Obtained as yellow crystals; yield: $1.47 \mathrm{~g}(62 \%)$; mp 211$213{ }^{\circ} \mathrm{C}$ (from acetic acid); IR (KBr) $v_{\max } / \mathrm{cm}^{-1}: 3082.9(\mathrm{CH}-$ aromatic), 2983.4 (CH-aliphatic), 2993.2 (CH-aliphatic), $1758.0(\mathrm{C}=\mathrm{O}$ ester $), 1653.9(\mathrm{C}=\mathrm{N}), 1591.7(\mathrm{C}=\mathrm{C}) ;{ }^{1} \mathrm{H}$ NMR (DMSO-d $) \delta$ 7.14-8.43 (m, 12H, ArH's), 6.36 (s, $1 \mathrm{H}$ pyrazoline), $4.03-4.14$ (q, $2 \mathrm{H}, J 7.0 \mathrm{~Hz}, \mathrm{COOCH}{ }_{2} \mathrm{CH}_{3}$ ), $0.91\left(\mathrm{t}, 3 \mathrm{H}, J 7.0 \mathrm{~Hz}, \mathrm{COOCH}_{2} \mathrm{CH}_{3}\right) ;{ }^{13} \mathrm{C}$ NMR (DMSO-d ${ }_{6}$ ) $\delta 165.17$ (d, J 256.1 Hz, C-F), 164.38 (C=O ester), 161.90 (d, J 256.3 Hz, C-F), 148.26, $146.25\left(\mathrm{C}=\mathrm{N}, \mathrm{C}-p-\mathrm{NO}_{2}\right)$, 143.43, 130.12 (d, $J 8.3 \mathrm{~Hz}, \mathrm{C}-m-\mathrm{F}), 129.20$ (d, $J 8.3 \mathrm{~Hz}$, C-m-F), 126.25 (d, J 3.1 Hz, C-p-F), 124.73, 124.62 (d, $J 3.1 \mathrm{~Hz}, \mathrm{C}-p-\mathrm{F}), 120.97,115.97$ (d, J $22.2 \mathrm{~Hz}, \mathrm{C}-o-\mathrm{F}$ ), $115.64(\mathrm{~d}, J 22.0 \mathrm{~Hz}, \mathrm{C}-o-\mathrm{F}), 111.02(\mathrm{C} \equiv \mathrm{N}), 70.92(\mathrm{C}-5$ pyrazoline), 63.35 (C-4 pyrazoline), $61.31\left(\mathrm{OCH}_{2} \mathrm{CH}_{3}\right)$, $12.91\left(\mathrm{OCH}_{2} \mathrm{CH}_{3}\right) ; \mathrm{MS}, \mathrm{m} / z: 476\left(\mathrm{M}^{+}, 13.7\right), 403(100.0), 357$ (22.7), 76 (10.2), 75 (7.2), 63 (8.0), 50 (6.0). Anal. Calc. for $\mathrm{C}_{25} \mathrm{H}_{18} \mathrm{~F}_{2} \mathrm{~N}_{4} \mathrm{O}_{4}\left(\mathrm{M}_{\mathrm{r}}=476.43\right): \mathrm{C}, 63.02 ; \mathrm{H}, 3.80 ; \mathrm{N}, 11.76 \%$; Found: C, 62.97; H, 3.85; N, $11.87 \%$.

Ethyl 5-cyano-3-(2,4-dichlorophenyl)-4-(4-fluorophenyl)1-(4-nitrophenyl)-2-pyrazoline-5-carboxylate 16e

Obtained as yellow crystals; yield: $1.55 \mathrm{~g}(59 \%)$; $\mathrm{mp}$ 210-212 ${ }^{\circ} \mathrm{C}$ (from dioxane - ethanol); IR (KBr) $v_{\max } / \mathrm{cm}^{-1}$ : 3069.3 (CH-aromatic), 2991.2 (CH-aliphatic), 1747.6 (C=O ester), $1595.1(\mathrm{C}=\mathrm{C}) ;{ }^{1} \mathrm{H}$ NMR (DMSO-d $)$ ) $7.11-8.42$ (m, $11 \mathrm{H}$, ArH's), 6.36 (s, 1H, 4-H pyrazoline), 4.08-4.19 (q, $\left.2 \mathrm{H}, J 7.2 \mathrm{~Hz}, \mathrm{COOCH} \mathrm{CH}_{3}\right), 0.92-0.99$ (t, $3 \mathrm{H}, J 7.2 \mathrm{~Hz}$, $\left.\mathrm{COOCH}_{2} \underline{\mathrm{CH}}_{3}\right) ;{ }^{13} \mathrm{C} \mathrm{NMR}$ (DMSO-d 6$) \delta 163.39(\mathrm{C}=\mathrm{O}$ ester $)$, $161.89(\mathrm{~d}, J 256.3 \mathrm{~Hz}, \mathrm{C}-\mathrm{F}), 148.58,146.13\left(\mathrm{C}=\mathrm{N}, \mathrm{C}-p-\mathrm{NO}_{2}\right)$,
143.83, 134.05, 133.26, 132.48, 130.37, $130.05(\mathrm{~d}, J 8.3 \mathrm{~Hz}$, C- $m$-F), 129.47, 126.88, 124.96, 124.53 (d, J3.2 Hz, C- $p$-F), $121.78,115.45(\mathrm{~d}, J 22.1 \mathrm{~Hz}, \mathrm{C}-o-\mathrm{F}), 112.98(\mathrm{C} \equiv \mathrm{N}), 70.71$ (C-5 pyrazoline), 64.25 (C-4 pyrazoline), $61.24\left(\mathrm{OCH}_{2} \mathrm{CH}_{3}\right)$, $12.84\left(\mathrm{OCH}_{2} \underline{\mathrm{CH}}_{3}\right) ; \mathrm{MS}, m / z: 528\left(\mathrm{M}^{+}+2,9.7\right), 526\left(\mathrm{M}^{+}, 15.4\right)$, 455 (68.9), 453 (100.0), 407 (23.5), 107 (10.1), 76 (10.7), 63 (12.1). Anal. Calc. for $\mathrm{C}_{25} \mathrm{H}_{17} \mathrm{Cl}_{2} \mathrm{FN}_{4} \mathrm{O}_{4}\left(\mathrm{M}_{\mathrm{r}}=527.33\right)$ : C, 56.93; H, 3.24; N, 10.62; Cl, 13.44\%; Found: C, 56.97; H, $3.25 ; \mathrm{N}, 10.60 ; \mathrm{Cl}, 13.46 \%$.

Ethyl 5-cyano-3-(4-fluorophenyl)-1,4-di-(4-nitrophenyl)2-pyrazoline-5-carboxylate $15 f$

Obtained as yellow crystals; yield: $1.33 \mathrm{~g} \mathrm{(53 \% );} \mathrm{mp}$ $176-178{ }^{\circ} \mathrm{C}$ (from acetic acid); IR (KBr) $v_{\text {max }} / \mathrm{cm}^{-1}: 3079.0$ (CH-aromatic), 2986.3 (CH-aliphatic), 1764.8 (C=O ester), 1654.5 (C=N), $1596.2(\mathrm{C}=\mathrm{C})$; ${ }^{1} \mathrm{H}$ NMR (DMSO-d $)$ ) 7.13 8.43 (m, 12H, ArH's), 6.36 (s, 1H pyrazoline), 4.09 (q, 2H, $J$ $\left.7.2 \mathrm{~Hz}, \mathrm{COOCH}{ }_{2} \mathrm{CH}_{3}\right), 0.90$ (t, $3 \mathrm{H}, J 7.2 \mathrm{~Hz}, \mathrm{COOCH}_{2} \underline{\mathrm{CH}}_{3}$ ); ${ }^{13} \mathrm{C} \mathrm{NMR}$ (DMSO-d ${ }_{6}$ ) $\delta 165.17$ (d, J $\left.256.1 \mathrm{~Hz}, \mathrm{C}-\mathrm{F}\right), 164.38$ $(\mathrm{C}=\mathrm{O}$ ester $), 148.26,146.25\left(\mathrm{C}=\mathrm{N}, \mathrm{C}-p-\mathrm{NO}_{2}\right), 143.43,129.20$ (d, $J 8.3 \mathrm{~Hz}, \mathrm{C}-m-\mathrm{F}), 127.25$ (d, $J 3.1 \mathrm{~Hz}, \mathrm{C}-p-\mathrm{F}), 124.73$, 120.97, 115.97 (d, J 22.2 Hz, C-o-F), $111.04(\mathrm{C} \equiv \mathrm{N}), 70.92$ (C-5 pyrazoline), 63.35 (C-4 pyrazoline), $61.31\left(\mathrm{OCH}_{2} \mathrm{CH}_{3}\right)$, $12.91\left(\mathrm{OCH}_{2} \underline{\mathrm{CH}}_{3}\right)$; MS, $m / z: 503\left(\mathrm{M}^{+}+1,17.4\right), 476$ (62.3), 430 (100.0), 384 (21.1), 76 (20.1), 63 (12.7), 50 (12.5). Anal. Calc. for $\mathrm{C}_{25} \mathrm{H}_{18} \mathrm{FN}_{5} \mathrm{O}_{6}\left(\mathrm{M}_{\mathrm{r}}=503.43\right)$ : $\mathrm{C}, 59.64 ; \mathrm{H}, 3.60 ; \mathrm{N}$, 13.91\%; Found: C, 59.49; H, 3.64; N, 13.87\%.

Synthesis of 1-aryl-3-(4-nitrophenyl)chromeno[3,4-c] pyrazol-4(3H)-ones 20

To a mixture of hydrazonoyl bromides $\mathbf{1 , 2}(5 \mathrm{mmol})$ and 3-cynocoumarin 19a or 3-phenylsulphonylcoumarin 19b or 3-bromocoumarine $19 \mathrm{c}(5 \mathrm{mmol})$, in dry benzene $(50 \mathrm{~mL})$ at room temperature, triethylamine $(0.7 \mathrm{~mL}, 5 \mathrm{mmol})$ was added. The mixture was refluxed till the hydrazonoyl bromide disappeared $(8 \mathrm{~h})$ as indicated by TLC analysis. After cooling to room temperature, the precipitated triethylamine hydrobromide was filtered and the solvent evaporated. Trituration of the residue with a small amount of methanol gave a crude solid. The latter was collected, washed with methanol and dried. Crystallization from suitable solvent gave the corresponding chromenopyrazoles 20a,b in good yield. The prepared compounds together with their physical and spectral data are listed below.

\section{1-(4-Fluorophenyl)-3-(4-nitrophenyl)chromeno[3,4-c] pyrazol-4(3H)-one $20 a$}

Obtained as brown solid; yield: $0.96 \mathrm{~g} \mathrm{(48 \% );} \mathrm{mp} \mathrm{310-}$ $312^{\circ} \mathrm{C}$ (from dioxane); IR (KBr) $v_{\max } / \mathrm{cm}^{-1}: 3089.6(\mathrm{CH}-$ aromatic), $1743.4(\mathrm{C}=\mathrm{O}$, lactone), $1657.8(\mathrm{C}=\mathrm{N}), 1605.0$ 
$(\mathrm{C}=\mathrm{C}) ;{ }^{1} \mathrm{H}$ NMR $\left(\mathrm{DMSO}_{-} \mathrm{d}_{6}\right) \delta$ 7.32-8.45 (12H, ArH's); ${ }^{13} \mathrm{C}$ NMR (DMSO-d ${ }_{6}$ ) $\delta 164.32$ (d, J 256.4 Hz, C-F), 156.16 (C=O lactone), 154.31 (C-O), 147.51, $146.24(\mathrm{C}=\mathrm{N}, \mathrm{C}-p-$ $\mathrm{NO}_{2}$ ), 142.40, 132.91, 131.29, 129.24 (d, J 8.3 Hz, C-m-F), 128.92, 126.81, 125.58 (d, J3.1 Hz, C-p-F), 125.17, 124.24, $121.62,116.12$ (d, J 22.2 Hz, C-o-F), 115.91, 112.15; MS, m/z: 401 (M+, 100.0), 355 (5.9), 257 (49.2), 211 (11.6), 187 (11.7), 163 (18.1), 136 (26.8), 123 (25.5), 90 (52.7), 76 (16.7), 75 (22.1), 63 (43.5), 50 (16.4). Anal. Calc. for $\mathrm{C}_{22} \mathrm{H}_{12} \mathrm{FN}_{3} \mathrm{O}_{4}\left(\mathrm{M}_{\mathrm{r}}=401.34\right): \mathrm{C}, 65.83 ; \mathrm{H}, 3.01 ; \mathrm{N}, 10.47 \%$; Found: C, 65.81; H, 3.00; N, 10.49\%.

\section{1-(2,4-Dichlorophenyl)-3-(4-nitrophenyl)chromeno[3,4-c] pyrazol-4(3H)-one $\mathbf{2 0 b}$}

Obtained as brown solid; yield: $1.15 \mathrm{~g} \mathrm{(51 \% );} \mathrm{mp} 232-$ $234^{\circ} \mathrm{C}$ (from acetic acid); IR (KBr) $v_{\max } / \mathrm{cm}^{-1}: 3085.3(\mathrm{CH}-$ aromatic), $1744.2(\mathrm{C}=\mathrm{O}$ lactone $), 1654.8(\mathrm{C}=\mathrm{N}), 1592.1$ $(\mathrm{C}=\mathrm{C}) ;{ }^{1} \mathrm{H}$ NMR $\left(\right.$ DMSO-d 6 ) $\delta 7.32-8.45\left(11 \mathrm{H}, \mathrm{ArH}^{\prime} \mathrm{s}\right) ;{ }^{13} \mathrm{C}$ NMR (DMSO-d $\left.{ }_{6}\right) \delta 156.16$ (C=O lactone), $154.31(\mathrm{C}-\mathrm{O})$, 147.51, $146.24\left(\mathrm{C}=\mathrm{N}, \mathrm{C}-p-\mathrm{NO}_{2}\right), 142.40,133.63,132.91$, $131.54,131.29,129.51,129.49,128.92,128.55,128.42$, 126.81, 125.17, 124.24, 121.62, 115.91, 112.17 (16C, ArC's); MS, m/z: $453\left(\mathrm{M}^{+}+2,61.0\right), 451\left(\mathrm{M}^{+}, 100.0\right), 206$ (11.3), 177 (14.5), 76 (46.7), 75 (49.9), 63 (19.6), 62 (21.5), 51 (13.8), 50 (48.3). Anal. Calc. for $\mathrm{C}_{22} \mathrm{H}_{11} \mathrm{Cl}_{2} \mathrm{~N}_{3} \mathrm{O}_{4}\left(\mathrm{M}_{\mathrm{r}}=\right.$ 452.24): C, 58.42; H, 2.45; N, 9.29; Cl, 15.67\%; Found: C, 58.17; H, 2.47; N, 9.39; Cl, 15.65\%.

Synthesis of 3a-acetyl(benzoyl)-1-aryl-3-(4-nitrophenyl)3a,9b-dihydrochromeno[3,4-c]-pyrazol-4(3H)-ones 24,25

This reaction was carried out by the same method described for the reaction of hydrazonoyl bromides $\mathbf{1 , 2}$ with 3-cyanocoumarin 19a using 3-acetylcoumarin 22 and 3-benzoylcoumarin $\mathbf{2 3}$ in place of 3-cyanocoumarin 19a. Crystallization from the suitable solvent gave the corresponding dihydrochromeno[3,4-c]pyrazole derivatives 24a,b and 25a,b respectively in good yield. The prepared compounds together with their physical and spectral data are listed below.

3a-Acetyl-1-(4-fluorophenyl)-3-(4-nitrophenyl)-3a,9bdihydrochromeno[3,4-c]-pyrazol-4(3H)-one $24 a$

Obtained as off white solid; yield: $1.13 \mathrm{~g}(51 \%) ; \mathrm{mp}$ 288-290 ${ }^{\circ} \mathrm{C}$ (from dimethylformamide-ethanol); IR (KBr) $v_{\max } / \mathrm{cm}^{-1}: 3107.7$ (CH-aromatic), 2985.6 (CH-aliphatic), 1734.8 $(\mathrm{C}=\mathrm{O}$ lactone $), 1712.9(\mathrm{C}=\mathrm{O}$ acetyl $), 1595.0(\mathrm{C}=\mathrm{C})$; ${ }^{1} \mathrm{H}$ NMR (DMSO-d $) \delta$ 7.14-8.31 (m, 11H, ArH's), 6.07 (s, 1H), 2.57 (s, 3H, $\mathrm{CH}_{3}$ acetyl); ${ }^{13} \mathrm{C}$ NMR (DMSO) $\delta$ 201.55 (C=O acetyl), 164.18 (d, J 256.1 Hz, C-F), 163.85 $(\mathrm{C}=\mathrm{O}$ lactone $), 150.87(\mathrm{C}-\mathrm{O}), 149.34,148.66(\mathrm{C}=\mathrm{N}$,
C-p- $\left.\mathrm{NO}_{2}\right), 140.70,130.17,130.84$ (d, J 8.3 Hz, C-m-F), 127.11, 125.21, 125.00, 124.57 (d, J 3.1 Hz, C-p-F), 116.90, 115.54 (d, J $22.2 \mathrm{~Hz}, \mathrm{C}-o-\mathrm{F}), 115.96,112.80,78.27$ (C-3 coumarin), 54.83 (C-4 coumarin), $25.10\left(\mathrm{CH}_{3}\right.$ acetyl); $\mathrm{MS}$, $m / z: 427$ ( $\left.\mathrm{M}^{+}-18\right), 401$ (100.0), 354 (14.1), 206 (11.5), 76 (30.2), 75 (21.8), 63 (13.0), 50 (20.6). Anal. Calc. for $\mathrm{C}_{24} \mathrm{H}_{16} \mathrm{FN}_{3} \mathrm{O}_{5}\left(\mathrm{M}_{\mathrm{r}}=445.39\right): \mathrm{C}, 64.71 ; \mathrm{H}, 3.62 ; \mathrm{N}, 9.43 \%$; Found: C, 64.70; H, 3.64; N, 9.42\%.

3a-Acetyl-1-(2,4-dichlorophenyl)-3-(4-nitrophenyl)-3a,9bdihydrochromeno [3,4-c]-pyrazol-4(3H)-one $\mathbf{2 4 b}$

Obtained as yellow solid; yield: $1.31 \mathrm{~g} \mathrm{(53 \% );} \mathrm{mp} \mathrm{196-}$ $197{ }^{\circ} \mathrm{C}$ (from acetic acid); IR (KBr) $v_{\max } / \mathrm{cm}^{-1}: 3107.7(\mathrm{CH}-$ aromatic), 2989.8 (CH-aliphatic), $1778.0(\mathrm{C}=\mathrm{O}), 1712.6$ $\left(\mathrm{C}=\mathrm{O}\right.$ acetyl), $1590.9(\mathrm{C}=\mathrm{C}) ;{ }^{1} \mathrm{H}$ NMR $\left(\right.$ DMSO-d $\left._{6}\right) \delta 7.12$ 8.27 (m, 11H, ArH's), 6.09 (s, 1H, 4-H coumarin), 2.57 (s, $3 \mathrm{H}, \mathrm{CH}_{3}$ acetyl); ${ }^{13} \mathrm{C} \mathrm{NMR}$ (DMSO) $\delta 201.53$ (C=O acetyl), 163.48 (C=O lactone), $150.68(\mathrm{C}-\mathrm{O}), 149.39,148.09(\mathrm{C}=\mathrm{N}$, C-p- $\left.\mathrm{NO}_{2}\right), 140.64,135.71,133.54,132.48,130.64,129.72$, 129.40, 127.72, 127.24, 125.41, 125.30, 116.86, 114.41, 112.52 (14C, ArC's), 79.19 (C-3 coumarin), 54.84 (C-4 coumarin), $25.02\left(\mathrm{CH}_{3}\right.$ acetyl); $\mathrm{MS}, \mathrm{m} / z: 497\left(\mathrm{M}^{+}+2,4.2\right)$, $495\left(\mathrm{M}^{+}, 6.7\right), 454$ (66.6), 453 (83.1), 452 (83.3), 451 (100.0), 409 (10.7), 408 (15.4), 407 (9.9), 406 (11.8), 373 (17.4), 178 (12.4), 76 (32.7), 50 (30.4). Anal. Calc. for $\mathrm{C}_{24} \mathrm{H}_{15} \mathrm{Cl}_{2} \mathrm{~N}_{3} \mathrm{O}_{5}$ $\left(\mathrm{M}_{\mathrm{r}}=496.29\right): \mathrm{C}, 58.08 ; \mathrm{H}, 3.04 ; \mathrm{N}, 8.46 ; \mathrm{Cl}, 14.28 \%$; Found: C, 57.72; H, 3.06; N, 8.58; Cl, 14.34\%.

3a-Benzoyl-1-(4-fluorophenyl)-3-(4-nitrophenyl)-3a,9bdihydrochromeno[3,4-c]-pyrazol-4(3H)-one 25a

Obtained as pale yellow solid; yield: $1.21 \mathrm{~g}(48 \%) ; \mathrm{mp}$ 271-273 ${ }^{\circ} \mathrm{C}$ (from dioxane); IR (KBr) $v_{\max } / \mathrm{cm}^{-1}: 3089.4$ (CH-aromatic), $1735.3(\mathrm{C}=\mathrm{O}), 1655.8$ (CO benzoyl), $1594.5(\mathrm{C}=\mathrm{C}) ;{ }^{1} \mathrm{H}$ NMR (DMSO-d $\left.{ }_{6}\right) \delta$ 7.15-8.13 (m, 17H, ArH's), 6.38 (s, 1H, 4-H coumarin); ${ }^{13} \mathrm{C}$ NMR (DMSO) $\delta 197.55$ (C=O benzoyl), 164.18 (d, $J 256.1 \mathrm{~Hz}, \mathrm{C}-\mathrm{F})$, $162.85(\mathrm{C}=\mathrm{O}$ lactone $), 150.92(\mathrm{C}-\mathrm{O}), 149.23,148.68(\mathrm{C}=\mathrm{N}$, C-p- $\left.\mathrm{NO}_{2}\right), 140.70,132.63,131.87,131.31,130.17,130.84$ (d, $J 8.3 \mathrm{~Hz}, \mathrm{C}-m-\mathrm{F}), 127.73,127.10,125.18,125.02$, 124.57 (d, $J 3.1 \mathrm{~Hz}, \mathrm{C}-p-\mathrm{F}$ ), 116.90, 116.27 (d, $J 22.2$ Hz, C-o-F), 115.96, 112.87, 78.27 (C-3 coumarin), 54.83 (C-4 coumarin); MS, m/z: $507\left(\mathrm{M}^{+}, 53.0\right), 401$ (11.9), 257 (100.0), 211 (12.6), 163 (20.0), 136 (28.2), 123 (66.7), 105 (20.9), 90 (39.3), 77 (34.7), 63 (25.1), 51 (16.1), 50 (13.4). Anal. Calc. for $\mathrm{C}_{29} \mathrm{H}_{18} \mathrm{FN}_{3} \mathrm{O}_{5}\left(\mathrm{M}_{\mathrm{r}}=507.46\right)$ : $\mathrm{C}, 68.63 ; \mathrm{H}$, 3.57; N, 8.28\%; Found: C, 68.60; H, 3.56; N, 8.27\%.

3a-Benzoyl-1-(2,4-dichlorophenyl)-3-(4-nitrophenyl)3a,9b-dihydrochromeno[3,4-c]-pyrazol-4(3H)-one $25 \boldsymbol{b}$

Obtained as yellow solid; yield: $1.31 \mathrm{~g}(47 \%)$; mp 193$195^{\circ} \mathrm{C}$ (from dioxane); IR (KBr) $v_{\max } / \mathrm{cm}^{-1}: 3072.0(\mathrm{CH}-$ 
aromatic), 1769.3 (br., C=O lactone, benzoyl), $1671(\mathrm{C}=\mathrm{N})$, $1586.1(\mathrm{C}=\mathrm{C}) ;{ }^{1} \mathrm{H}$ NMR $\left(\right.$ DMSO-d $\left._{6}\right) \delta$ 7.13-8.24 (m, 16H, ArH's), 6.36 (s, 1H, 4-H coumarin); ${ }^{13} \mathrm{C}$ NMR (DMSO) $\delta 197.53(\mathrm{C}=\mathrm{O}$ benzoyl), $162.85(\mathrm{C}=\mathrm{O}$ lactone $), 150.52$ (C-O), 149.20, 148.13 (C=N, C- $\left.p-\mathrm{NO}_{2}\right), 140.72,135.69$, 133.31, 132.91, 132.66, 131.76, 131.30, 130.81, 129.76, $129.68,127.96,127.70,126.87,125.32,125.12,116.93$, 115.82, 112.73 (18C, ArC's), 78.27 (C-3 coumarin), 54.83 (C-4 coumarin); MS, $m / z: 559\left(\mathrm{M}^{+}+2,3.2\right), 557\left(\mathrm{M}^{+}, 10.1\right)$, 453 (66.2), 451 (100.0), 405 (11.7), 307 (8.3), 206 (15.5), 178 (18.0), 177 (19.1), 151 (18.9), 105 (30.1), 76 (75.4), 75 (83.8), 63 (31.2), 51 (31.9), 50 (79.1). Anal. Calc. for $\mathrm{C}_{29} \mathrm{H}_{17} \mathrm{Cl}_{2} \mathrm{~N}_{3} \mathrm{O}_{5}\left(\mathrm{M}_{\mathrm{r}}=558.36\right): \mathrm{C}, 62.37 ; \mathrm{H}, 3.06 ; \mathrm{N}, 7.52 ; \mathrm{Cl}$, $12.69 \%$; Found: C, 62.38; H, 3.06; N, 7.51; Cl, 12.68\%.

Oxidation of chromeno[3,4-c]pyrazol-4(3H)-one derivatives 24 and 25

\section{General procedure}

A suspension of the products $\mathbf{2 4} \mathbf{a}$ or $\mathbf{2 5} \mathbf{a}(5 \mathrm{mmol})$ in aqueous potassium hydroxide $(10 \mathrm{~mL}, 10 \%)$ was refluxed for $12 \mathrm{~h}$. The reaction mixture was cooled, poured into water $(50 \mathrm{~mL})$ and acidified with hydrochloric acid $\left(4 \mathrm{~mL}, \mathrm{~mol} \mathrm{~L}^{-1}\right)$. The crude product was filtered, washed with water, dissolved in toluene $(10 \mathrm{~mL})$ and refluxed for $2 \mathrm{~h}$. After cooling, the product that precipitated was collected and crystallized from suitable solvent. The pure products were identical in all aspects ( $\mathrm{mp}$, mixed $\mathrm{mp}$ and spectroscopic data) with 20a. Similarly, oxidation of 24b or $\mathbf{2 5 b}$ gave compound $\mathbf{2 0 b}$.

\section{Results and Discussion}

1,3-Dipolar cycloaddition of nitrilimines $\mathbf{3 , 4}$, prepared in situ from hydrazonyl bromides $\mathbf{1 , 2}$ in dry benzene in the presence of triethylamine, to $\alpha$-cyanocinnamonitriles $\mathbf{5 a - f}$ was carried out at reflux for $8 \mathrm{~h},{ }^{24}$ and gave exclusively 3,4-diaryl-1-(4-nitrophenyl)pyrazole-5-carbonitriles 8a-e, 9a-f respectively (Scheme 1).

The intermediate pyrazolines $\mathbf{6 , 7}$ were not detected in any case. The structures of the isolated products 8,9 were elucidated by their elemental analyses and spectroscopic data. The ${ }^{1} \mathrm{H}$ NMR revealed, in each case, the absence of signals assignable to the 4- $\mathrm{CH}$ and $5-\mathrm{CH}$ protons of the corresponding pyrazoline derivatives 6,7 and $10,11^{32}$ and the IR spectra of the products $\mathbf{8 , 9}$ show a nitrile absorption band at about 2224-2235 $\mathrm{cm}^{-1}$. This finding suggests that the 5,5-dicyano-2-pyrazoline derivatives $\mathbf{6 , 7}$ are easily aromatized by thermal elimination of hydrogen cyanide to give 8,9. Such elimination is analogous to the thermal elimination of hydrazoic acid ${ }^{33}$ from 5-azido-5-benzoyl- 1,3,4-triphenyl-2-pyrazolin and of benzenesulfinic acid ${ }^{32}$ from 5-benzenesulfonyl-1,3,4-triphenyl-2-pyrazoline.

The signals of ${ }^{13} \mathrm{C}$ NMR spectra of $\mathbf{8 b}$ and $9 \mathbf{c}$ are compatible with the proposed structure. Thus, they display a signal of methyl carbon at 20.39 and of methoxy carbon at $54.75 \mathrm{ppm}$. The $\mathrm{C} \equiv \mathrm{N}$ signals appear at 110.58 and 110.78 ppm respectively.

The regiochemistry of $\mathbf{8 , 9}$ was confirmed by comparison of the properties of $\mathbf{8} \mathbf{a}$ with the pertinent regioisomer 3-(4-fluorophenyl)-1-(4-nitrophenyl)-5-phenylpyrazole4-carbonitrile 7, which was prepared from the reaction of 1 with phenacyl cyanide. ${ }^{34}$

Similarly, the reaction of 1,2 with $\alpha$-cyanocinnamamide 12a,b in benzene at reflux temperature gave the corresponding pyrazole derivatives 13a,b (Scheme 1). Like the previous reaction, thermal elimination of hydrogen cyanide took place. The structures of the products were confirmed considering the correct elemental analyses and spectroscopic data (Experimental part).

The ${ }^{1} \mathrm{H}$ NMR spectra of the products $\mathbf{1 3}$ showed no signal due to 4-CH characteristic of 2-pyrazoline derivative (Experimental part). This result confirms the elimination of hydrogen cyanide.

The reaction of the ethyl $\alpha$-cyanocinnamates 14a-f with the 1,2 when carried out in a similar manner, gave the corresponding 2-pyrazoline derivatives $\mathbf{1 5 , 1 6}$ (Scheme 2).

The thermal elimination of hydrogen cyanide from the reaction products 15,16 was not observed. The structure of the products was in agreement with their elemental analyses and spectroscopic data. Although compounds 15,16 bear a nitrile group its IR absorption band does not appear similar to the case of aliphatic nitriles activated by a nitrogen or oxygen atom in the $\alpha$-position. ${ }^{35,36}$ This similarity of the absence of the nitrile absorption in the IR spectra together with the chemical shift value $6.2 \mathrm{ppm}$ observed for the methine proton also exclude the possibility of the other regioisomer $\mathbf{1 7 , 1 8}$ for the isolated product. This is because compounds of type $\mathbf{1 7 , 1 8}$ are expected to exhibit strong nitrile absorption in their IR spectra ${ }^{37}$ and their methine chemical shift at the 5-position would appear at upper field $(5.1 \mathrm{ppm}) .^{38}$ The decisive evidence for the existence of the nitrile group is provided by the ${ }^{13} \mathrm{C}$ NMR of compounds $\mathbf{1 5}, \mathbf{1 6}$. Undoubtedly the signals at $112.02-112.98 \mathrm{ppm}$ are attributed to nitrile carbon atoms. The mass spectra of compounds 15,16 show the correct molecular ions and the most important fragmentation pathways of the molecular ion involve generation of $\left[\mathrm{M}-73\left(\mathrm{COOC}_{2} \mathrm{H}_{5}\right)\right]^{+}$ions (base peaks). Another common fragment results from loss of $\mathrm{NO}_{2}$ and give $[\mathrm{M}-73-46]^{+}$ions.

Also, the reaction of 3-cyanocoumarin 19a with hydrazonoyl bromides $\mathbf{1 , 2}$ in refluxing benzene in the 


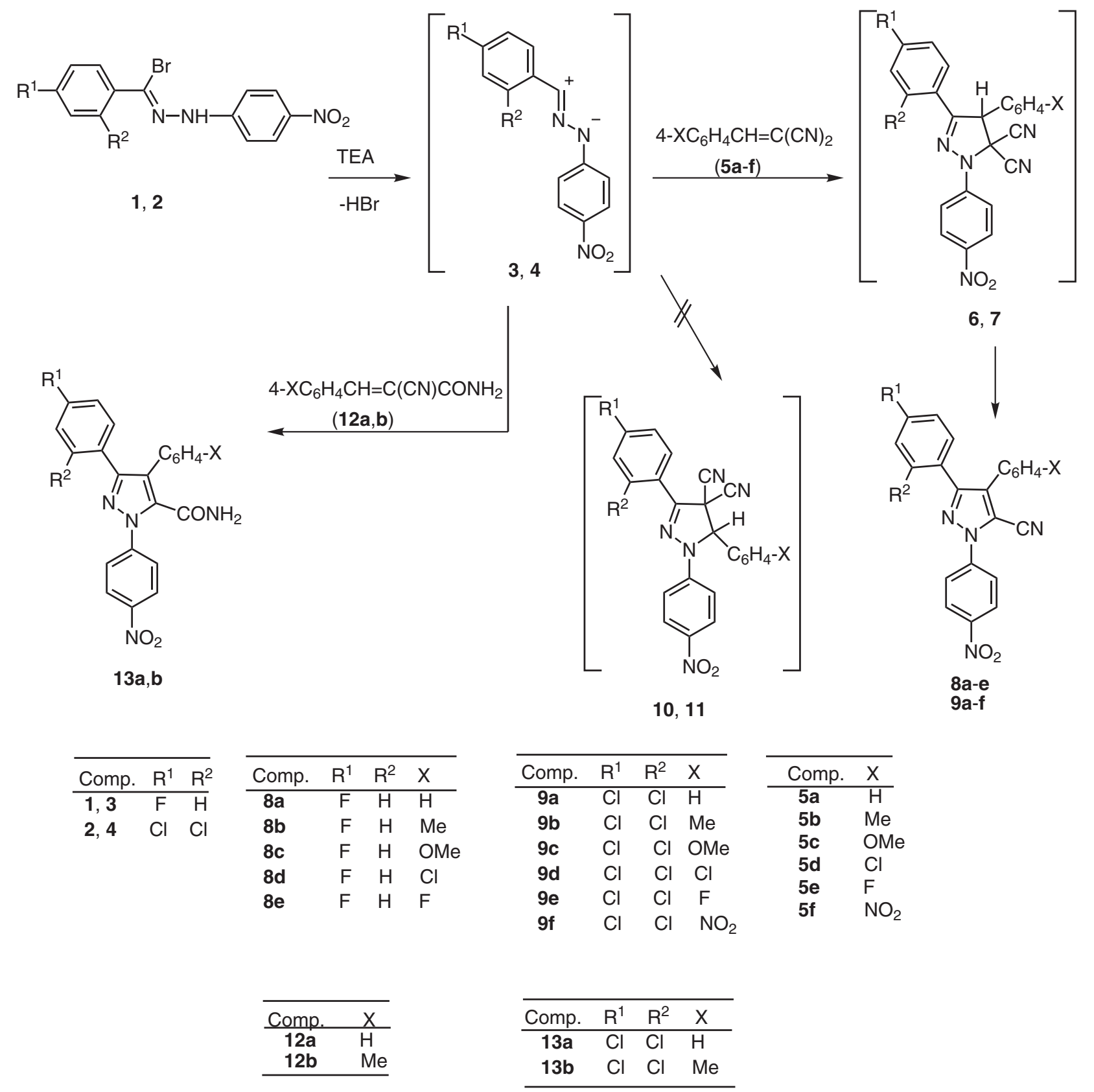

Scheme 1.

presence of triethylamine gave only one isolable product in each case 20a and 20b which analyzed correctly for the proposed structures. The ${ }^{1} \mathrm{H}$ NMR spectra of 20a,b revealed the absence of the methine $9 \mathrm{~b}$ hydrogen in the cycloadducts 21a,b (Scheme 3) and the IR spectrum showed the absence of the cyano group absorption band. These results indicate that the cycloadducts 21a,b undergo simultaneous elimination of hydrogen cyanide as soon as it is formed to give 1-aryl-3-(4-nitrophenyl)chromeno[3,4-c] pyrazol-4(3H)-one derivatives 20a,b.
The products 20a,b were also formed via cycloaddition of the nitrilimines $\mathbf{3 , 4}$ to 3-phenylsuphonylcoumarin 19b or 3-bromocoumarin 19c. The products 20a,b result undoubtedly via thermal elimination of benzenesulfinic acid and hydrogen bromide from the corresponding cycloadducts (Scheme 3).

The reaction of hydrazonoyl bromides $\mathbf{1 , 2}$ with acetylcoumarin $\mathbf{2 2}$ and benzoylcoumarin $\mathbf{2 3}$ in refluxing benzene in the presence of triethylamine afforded the 1,3-dipolar cycloadducts 1-aryl-3-(4-nitrophenyl)-3aR- 
<smiles>[R]c1ccc(/[C+]=N/[N-]c2ccc([N+](=O)[O-])cc2)c([R7])c1</smiles>

3, 4
4- $\mathrm{XC}_{6} \mathrm{H}_{4} \mathrm{CH}=\mathrm{C}(\mathrm{CN}) \mathrm{COOEt}$ (14a-f)

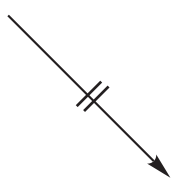

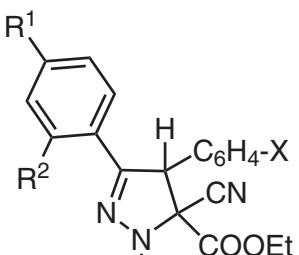<smiles>Cc1ccc([N+](=O)[O-])cc1</smiles>

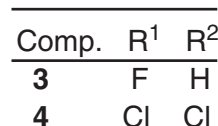<smiles>O=C(I)c1cc2ccccc2oc1=O</smiles>

22,23

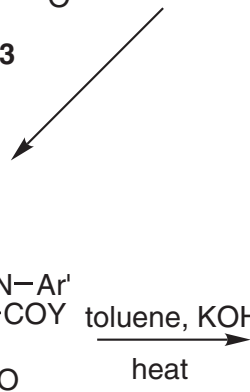

$24 a, b$

$25 a, b$<smiles>O=C1Oc2ccccc2C2C(Br)=NN([Al])C12O</smiles>

\begin{tabular}{ccll}
\hline Comp. & $\mathrm{R}^{1}$ & $\mathrm{R}^{2}$ & $\mathrm{X}$ \\
\hline $15 \mathrm{a}$ & $\mathrm{F}$ & $\mathrm{H}$ & $\mathrm{H}$ \\
$15 \mathrm{~b}$ & $\mathrm{~F}$ & $\mathrm{H}$ & $\mathrm{Me}$ \\
$15 \mathrm{c}$ & $\mathrm{F}$ & $\mathrm{H}$ & $\mathrm{OMe}$ \\
$15 \mathrm{~d}$ & $\mathrm{~F}$ & $\mathrm{H}$ & $\mathrm{Cl}$ \\
$15 \mathrm{e}$ & $\mathrm{F}$ & $\mathrm{H}$ & $\mathrm{F}$ \\
$15 \mathrm{f}$ & $\mathrm{F}$ & $\mathrm{H}$ & $\mathrm{NO}_{2}$
\end{tabular}

\begin{tabular}{llll}
\hline Comp. & $\mathrm{R}^{1}$ & $\mathrm{R}^{2}$ & $\mathrm{X}$ \\
\hline $16 \mathrm{a}$ & $\mathrm{Cl}$ & $\mathrm{Cl}$ & $\mathrm{H}$ \\
$16 \mathrm{~b}$ & $\mathrm{Cl}$ & $\mathrm{Cl}$ & $\mathrm{Me}$
\end{tabular}

16c $\mathrm{Cl} \mathrm{Cl} \mathrm{OMe}$

16d $\mathrm{Cl} \quad \mathrm{Cl} \mathrm{Cl}$

16e $\mathrm{Cl} \mathrm{Cl} \mathrm{F}$

Scheme 2.<smiles>[X]CCCC1(C(=O)OCC)N(c2ccc([N+](=O)[O-])cc2)N=C(c2ccc([R7])cc2[R])C1(C#N)C(=O)OCC</smiles>

17,18<smiles></smiles>

3, 4<smiles>O=c1oc2ccccc2cc1Cl</smiles>

19a-c

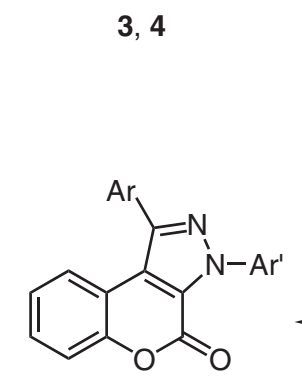

20a, b

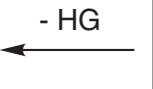<smiles>O=C1Oc2ccccc2C2C(Br)=NN(Br)C12Cl</smiles>

21

\footnotetext{
$\mathrm{a}: \mathrm{Ar}=4-\mathrm{FC}_{6} \mathrm{H}_{4} ; \mathrm{b}: \mathrm{Ar}=2,4-\mathrm{Cl}_{2} \mathrm{C}_{6} \mathrm{H}_{3}$;

$A r^{\prime}=4-\mathrm{NO}_{2} \mathrm{C}_{6} \mathrm{H}_{4}$
}

G: 19a, CN; 19b, $\mathrm{SO}_{2} \mathrm{Ph} ; 19 \mathrm{c}, \mathrm{Br}$

22, 24, $\mathrm{Y}=\mathrm{CH}_{3}$

23, 25, $\mathrm{Y}=\mathrm{Ph}$

Scheme 3. 
3a,9b-dihydrochromeno[3,4-c]pyrazole-4(3H)-ones 24a,b and 25a,b respectively (Scheme 3 ). The assigned structures $\mathbf{2 4 a}, \mathbf{b}$ and $\mathbf{2 5 a}, \mathbf{b}$ were supported by analytical and spectroscopic data (Experimental part). In their ${ }^{1} \mathrm{H}$ NMR spectra, they have characteristic signals due to $9 \mathrm{~b}$ proton resonance near 6.0 and $6.3 \mathrm{ppm}$, respectively. The chemical shifts seem to be compatible with the assigned structures 24a,b and 25a,b and exclude the possibility of the other regioisomer. The ${ }^{13} \mathrm{C}$ NMR of compound $\mathbf{2 4 b}$ taken as example, displays two characteristic signals of carbonyl carbon of acetyl group and lactone at 201.53 and 163.48 ppm respectively. The signals at 79.19, 54.84 and 25.02 are attributed to C-3a, C-9b and methyl carbon of acetyl group. The structures of the products were also confirmed by their conversion to $\mathbf{2 0 a}, \mathbf{b}$. Thus, by refluxing $\mathbf{2 4 a}, \mathbf{b}$ or $\mathbf{2 5 a}, \mathbf{b}$ in aqueous potassium hydroxide (10\%) followed by heating the crude product in toluene gave, in both cases, 20a,b (Scheme 3).

\section{Biological activity}

Antibacterial and antifungal screening was carried out using the agar diffusion technique. ${ }^{39}$ Most of the newly synthesized compounds were tested for their antibacterial activity in vitro against several pathogenic bacterial strains such as gram-negative, Escherichia coli, Enterobacter aerogenes, Pseudomonas aeruginosa, Klebsiella oxytocca, gram-positive, Staphylococcus aureus, and their antifungal activity against the fungi Candida albicans and Aspergillus flavus at a concentration $200 \mu \mathrm{g} \mathrm{mL}^{-1}$ using DMSO as a solvent. DMSO showed no inhibition zone. Erythromycin (Himedia, India, LOT NO.0000008821), Cephalexin (Himedia, India, LOT NO.0000010511), Tetracycline, (Himedia, India, LOT NO.0000014267) and Flucoral were used as reference substances. The results are illustrated in Table 1 as average diameter of inhibition zone in $\mathrm{mm}$.

As shown in the table most of the tested pyrazoles and pyrazolines showed low to moderate activities against $E$. coli. Against $P$. aeruginosa the pyrazole derivatives were found to have higher activity than pyrazoline derivatives. It is noticed that only pyrazoline derivatives possess moderate to high activity against $S$. aureus, and among them the compounds have fluorophenyl group at position 3 of pyrazole or at position 1 of chromeno[3,4-c]pyrazole were more effective than those that have dichlorophenyl group. Also,

Table 1. Antimicrobial screening result of the tested compounds

\begin{tabular}{|c|c|c|c|c|c|c|c|}
\hline \multicolumn{8}{|c|}{ Inhibition zone diameter (mm per $200 \mathrm{mcg}$ sample) } \\
\hline Compound & C. albicans & A. flavus & E. Coli & P. aeruginosa & E. aerogenes & S. aureus & K. oxytoca \\
\hline DMSO & - & - & - & - & - & - & - \\
\hline $8 \mathbf{a}$ & - & - & 11 & 13 & - & - & 12 \\
\hline 9a & - & - & - & 14 & - & - & - \\
\hline $9 \mathrm{~b}$ & - & - & 14 & - & - & - & - \\
\hline $8 c$ & - & 12 & 12 & 8 & 6 & - & 7 \\
\hline $8 d$ & - & - & 12 & 13 & - & - & 11 \\
\hline 9d & - & - & - & 13 & - & - & - \\
\hline $8 \mathrm{e}$ & - & - & 15 & - & - & - & 13 \\
\hline $9 e$ & - & - & 12 & 14 & - & - & - \\
\hline 9f & - & - & - & - & - & - & - \\
\hline $15 b$ & 9 & - & 13 & 9 & - & 18 & 14 \\
\hline $16 \mathrm{~b}$ & - & - & 16 & - & - & 11 & 14 \\
\hline 16d & 12 & - & 12 & 8 & 11 & - & - \\
\hline $15 e$ & 8 & - & 14 & 9 & - & 14 & 14 \\
\hline $16 e$ & - & - & - & - & - & 12 & 13 \\
\hline $15 f$ & 11 & - & - & 12 & 10 & 16 & 12 \\
\hline $20 \mathrm{a}$ & 14 & - & 8 & - & 9 & - & 12 \\
\hline $20 \mathrm{~b}$ & - & - & 12 & - & - & 11 & - \\
\hline $24 b$ & 8 & - & 12 & - & - & - & 12 \\
\hline $25 a$ & 10 & - & 8 & - & - & 19 & - \\
\hline $25 b$ & 13 & - & 11 & - & 6 & - & - \\
\hline Erythromycin ${ }^{\mathrm{a}}$ & - & - & 14 & 15 & 13 & 12 & 30 \\
\hline Cephalexin $^{\mathrm{b}}$ & - & - & 15 & - & 14 & 15 & 25 \\
\hline Tetracyclin ${ }^{\mathrm{b}}$ & - & - & 18 & - & 15 & 15 & 20 \\
\hline Flucoral & 16 & 14 & - & - & - & - & - \\
\hline
\end{tabular}

${ }^{\mathrm{a}} 15 \mu \mathrm{g} \mathrm{mL}{ }^{-1}$; ${ }^{\mathrm{b}} 30 \mu \mathrm{g} \mathrm{mL}{ }^{-1}$. 
the activity against $C$. albicans and $E$. aerogenes was noticed for pyrazolines and fused pyrazolines rather than pyrazoles or fused pyraozles. On the other hand, most of the tested pyrazoles and pyrazolines exhibited moderate activity against $K$. oxytoca, but it is obvious that among pyrazole derivatives, the active compounds were the fluorinated ones. None of the tested compounds except $\mathbf{8 c}$ inhibits the growth of A. flavus. Compound $9 \mathbf{f}$ showed no inhibitory effect against any of the tested organisms. It is important to mention that compound 9f is the only pyrazole derivative that have nitro group on phenyl substituent at position 4 .

\section{Conclusions}

Different pyrazole derivatives, as well as pyrazoline derivatives and their chromenofused derivatives were synthesized, completely characterized and evaluated for their antibacterial and antifungal activities.

The test results have evidenced that the pyrazoline and fused pyrazoline compounds have higher activities than the pyrazole and fused pyrazole ones.

Concerning the substitution in pyrazole $\mathbf{8 a}, \mathbf{8 c}, \mathbf{8 d}, \mathbf{8 e}$, 15b, 15e, 15f and pyrazoline compounds 9a, 9b, 9d, 9e, 9f, 16b, 16e at the phenyl group in 3-position demonstrated that the fluorosubstituted compounds are more effective than the chlorosubstituted analogues, and compound 16d was exception to this trend.

\section{References}

1. Sado, T.; Inoue, A.; Jpn. Kokai Tokkyo Koho 901010781990. (CA 113: 78422k)

2. Bekhit, A. A.; Ashour, H. M. A.; Abdel Ghany, Y. S.; Bekhit, A. E. A.; Baraka, A.; Eur. J. Med. Chem. 2008, 43, 456.

3. Bekhit, A. A.; Abdel-Aziem, T.; Bioorg. Med. Chem. 2004, 12, 1935.

4. Akahane, A.; Kuroda, S.; Itani, I.; Tabuchi, S.; Sato, Y.; Matsuoda, N.; Tada, M.; Matsuka, H.; Oku, T.; Tanaka, A.; PCT Int. Appl. 69 pp. CODEN: PIXXD2 WO $2001040230 \mathrm{Al}$ 20010607, 2001. (CA 135: 33489a).

5. Akahane, A.; Minagawa, M.; Tanaka, A.; (Fujisawa Pharmaceutical Co., Ltd Japan) A. PCT Int. Appl. 63 pp. Pub. No.: WO 2003045950 A1 232003 2002, (CA 138: 24732a).

6. Int. Appl., 34 pp. CODEN: P1XXD2 WO 2002100864 A1 20021219 Designated States, 2002; Takabe, F.; Shibayama, A.; Yamaguchi, M.; Yamaji, M.; Hanai, R.; Sadohara, H.; Jpn. Kokai Tokkyo Kono 9759276 1997. (CA 126: 277477a).

7. El-Emary, T. I.; El-Dean, A. M. K; El-Kashef, H. S.; Il Farmaco 1998, 53, 383.

8. El-Kashef, H. S.; El-Emary, T. I.; Gasquet, M.; Timon-David, P.; Maldonado, J.; Vanelle, P.; Pharmazie 2000, 55, 572.
9. Korgaokar, S. S.; Patil, P. H.; Shah, M. J; Parekh, H. H.; Indian J. Pharm. Sci. 1996, 58, 222.

10. Palaska, E.; Aytemir, M.; Uzbay, IT.; Erol, D.; Eur. J. Med. Chem. 2001, 36, 539.

11. Rajendra, P. Y.; Lakshmana, R. A.; Prasoona, L.; Murali, K.; Ravi, K. P.; Bioorg. Med. Chem. Lett. 2005, 15, 5030.

12. Ozdemir, Z.; Kandilici, H. B.; Gumusel, B.; Calis, U.; Bilgin, A A.; Eur. J. Med. Chem. 2007, 42, 373.

13. Ruhogluo, O.; Ozdemir, Z.; Calis, U.; Gumusel, B.; Bilgin, A A.; Arzneimittelforschung 2005, 55, 431.

14. Udupi, R. H.; Kushnoor, A. S.; Bhat, A. R.; Indian J. Heterocycl. Chem. 1998, 8, 63.

15. Nauduri, D.; Reddy, G. B.; Chem. Pharm. Bull. (Tokyo) 1998, 46, 1254.

16. Taylor, E. C.; Patel, H. H.; Tetrahedron 1992, 48, 8089.

17. El-Saghier, A. M. M.; Naili, M. B.; Rammash, B. Kh.; Saleh, N. A.; Kreddan, K. M.; Arkivoc 2007, xvi, 83.

18. Koga, H.; Itoh, A.; Murayama, S.; Suzue, S.; Irikura, T.; J. Med. Chem. 1980, 23, 1358.

19. Wilkinson, J. A.; Chem. Rev. 1992, 92, 505.

20. Wise, R.; Andrews, J. M.; Edwards, L. J.; Antimicrob. Agents Chemother. 1983, 23, 559.

21. Hagen, S. E.; Domagala, J. M.; Heifetz, C. L.; Johnson, J.; J. Med. Chem. 1991, 34, 1155.

22. Matsumoto, J.; Miyamoto, T.; Minamada, A.; Mishimuna, Y.; Egawa, H.; Nisimira, H.; J. Med. Chem. 1984, 27, 292.

23. Hayakawa, I.; Hiramitsu, T.; Tanaka, Y.; Chem. Pharm. Bull. (Tokyo), 1984, 32, 4907.

24. Hassaneen, H. M; Shawali, A. S.; Elwaln, N. M.; Abunada, N. M.; Algharib, M. S.; Arch. Pharmacal Res. 1992, 15, 292.

25. Hassaneen, H. M.; Hilal, R. H.; Elwan, N. M.; Harhash, A.; Shawali, A. S.; J. Heterocycl. Chem. 1984, 21, 1013.

26. Hassaneen, H. M.; Mousa, H. A. H.; Shawali, A. S.; J. Heterocycl. Chem. 1987, 24, 1665.

27. Hassaneen, H. M.; Mousa, H. A. H.; Abed, N. M.; Shawali, A. S.; Heterocycles 1988, 27, 695.

28. Hassaneen, H. M.; Harhash, A. E.; Abunada, N. M.; Abdallah, T. A.; Algharib, M. S.; J. Chem. Res. 1993, 194.

29. Abunada, N. M.; Hassaneen, H. M.; Jordan J. Chem. 2008, 3, 1.

30. Hegarty, A. F.; Scott, F. L.; J. Chem. Soc. 1966, 672.

31. Shawali, A. S.; Hassaneen, H. M.; Tetrahedron 1972, 29 , 121.

32. Shimizu, T.; Hayashi, Y.; Miki, M.; Teramura, K.; J. Org. Chem. 1985, 50, 904.

33. L'abbe, G. L.; Mathys, G.; J. Heterocycl. Chem. 1974, 11, 613.

34. Abunada, N. M.; Hassaneen, H. M.; Kandile, N. G.; Miqdad, O. A.; Molecules 2008, 13, 1501.

35. Butt, G.; Cilmi, J.; Hooben, P. M.; Topson, R. D.; Spectrochim. Acta 1980, 36, 521. 
36. Jesson, J. P.; Thompson, H. W.; Spectrochim. Acta 1958, 13, 217.

37. Thomas, B. H.; Orville-Thomas, W. J.; J. Mol. Struct. 1971, 7 , 123.
38. Shimizu, T.; Hayashi, Y.; Nishio, T.; Teramura, K.; Bull. Chem. Soc. Jpn. 1984, 57, 787.

39. Bauer, A. W.; Kirby, W. M. M.; Sgerris, J. C.; Turck, M.; Am. J. Clin. Path. 1966, 45, 493.

Received: July 21, 2008

Web Release Date: April 30, 2009 